Periodica Polytechnica Civil Engineering, 65(2), pp. 619-637, 2021

\title{
Technological Aspects of Usage of Calcareous Fly Ash as a Main Constituent of Cements
}

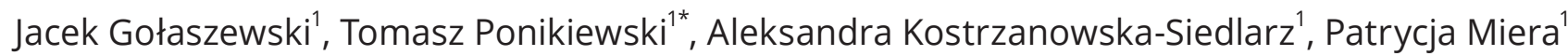 \\ ${ }^{1}$ Department of Processes Engineering and Building Physics, Faculty of Civil Engineering, Silesian University of Technology, 44-100 \\ Gliwice, Akademicka 5 St., Poland \\ * Corresponding author, e-mail: tomasz.ponikiewski@polsl.pl
}

Received: 20 June 2017, Accepted: 01 May 2020, Published online: 16 February 2021

\begin{abstract}
The use of mineral additives allows you to modify the properties of concrete and result in substantial economic benefits. The research the influence cement type with calcareous fly ash (W) content in cement, method of cement production, activation by grinding calcium fly ash and batch of fly ash on the rheology, plastic shrinkage, air content, the setting time and heat of hydration of mortar are presented in this paper. The results show that cements produced by intergrinding of the constituents or blending with fly ash preprocessed by milling, are characterized by acceptable technological properties, not differing significantly from other currently used cements. It is not recommended to use cements obtained by blending with raw calcareous fly ash W. Calcareous fly ash used for the production of cement should be selected because of its properties. According to conducted tests, this criterion can be the volume density of the ash, which should be at least $900 \mathrm{~kg} / \mathrm{m}^{3}$. The negative effect of calcareous fly ash used as an additive for cement on workability is considerably smaller than when it is used as an additive type II.
\end{abstract}

Keywords

calcareous fly ash, cement mortars, rheological properties, setting time, air content, heat of hydration, plastic shrinkage

\section{Introduction}

Mineral admixtures play a significant part in the modern concrete technology - its use is one of the main directions for cement and concrete technology progress; being at the same time an important element of a sustainable development strategy. By using the mineral admixtures beneficial effects can be obtained: technical (in respect to concrete durability), economical (in respect to whole live cycle of construction) and ecological: reduction of energy consumption (due to reduction of cement or/and clinker content in cement) and lowering the $\mathrm{CO}_{2}$ emission. Mineral additives can be applied directly to the concrete or as the main ingredient cement. Currently, standard [1] provides for the possibility of use as additives siliceous and calcareous fly ashes (V, W), ground granulated blast-furnace slag $\mathrm{S}$, natural and industrial pozzolans (P, Q), silica fume (D), the burnt shale $(\mathrm{T})$ and limestone (L, LL). Out of the aforementioned mineral additives most commonly used are $\mathrm{S}$, V, L and LL. Effects of these admixtures on the properties of cement, fresh and hardened concrete has been widely examined and described for example, in the [2-5].
Calcareous fly ash (W) is produced as a result of burning brown coal in conventional furnaces in large amount - in Poland about 5 million tons every year of $\mathrm{W}$ is produced [6]. Requirements for the calcareous fly ash (W) as the main and secondary component of common cements are defined in [6] and presented in Table 1, dry fly ashes from boiler furnaces are allowed.

Table 1 Requirements for calcareous fly ash (W) used in cement production

\begin{tabular}{lcc}
\hline Property & Requirements & $\begin{array}{c}\text { Calcareous fly ash } \\
\text { (W) Bełchatów }\end{array}$ \\
\hline & $<5,0 \%$ & \\
Loss of ignition & $<7,0 \%$ & 3.7 \\
& $<9,0 \%$ & 21 \\
Reactive $\mathrm{CaO}$ & $>10 \%$ & 31 \\
$\begin{array}{l}\text { Reactive } \mathrm{SiO}_{2} \\
\text { Hydraulic activity }-\end{array}$ & $>25 \%$ & - \\
$\begin{array}{l}\text { compressive strength of fly ash } \\
\text { mortar in acc. acc. with [1] }\end{array}$ & $\geq 10 \mathrm{MPa}$ & \\
Soundness, mm & $\leq 10 \mathrm{~mm}$ & $2 \mathrm{~mm}$ \\
\hline
\end{tabular}


Tested Calcareous fly ashes (W) from Bełchatów power plant demonstrates high sieve residue $(0.045 \mathrm{~mm})$ what results in significant water demand and problems with concrete workability control [7, 8]. Performed researches shown that worsening of the workability is directly proportional to the amount of fly ash (W) added [9-14] and increases with sieve residue increase [8]. Negative influence of fly ash (W) on workability of fresh concrete is the problem which considerably reduces the attractiveness of fly ash (W) use in concrete technology [15]. It was shown in $[7,8,13,15-18]$ that negative influence of fly ash (W) on the workability may be reduced by processing it by grinding, blending or separation. Favored solution is the production of cement with fly ash (W) as a main constituent - grinding is a routine technological process in cement production [7, 8]. Another possibility is production of composite cements containing mix of calcareous (W) and siliceous (V) fly ashes, granulated blast furnace slag (S) and limestone (L, LL). Due to lower water demand these mineral admixtures may partially or even totally eliminate negative influence of fly ash (W) on workability.

Up to date fly ashes (W) are used as cement additive to a limited extent. One of the main problems is a relatively small number of systematic research regarding fly ashes (W) influence on cement and concrete properties available. In order to check the possibilities and conditions for the efficient use of the fly ashes (W) in concrete technology was carried out wide and systematic research program, the results of which are shown in the [19, 20]. It has been shown that the presence of fly ashes (W) in cement usually does not adversely affect the mechanical properties and durability of hardened concrete and sometimes it even improves them [21-26]. What remains in accordance with the different research [12-14].

The main goal of presented research was to examine usability of cements containing calcareous fly ash (W) from technological point of view. In a broader aspect, the research contributes to popularize possibility of calcareous fly ash use in cement and concrete technology, what greatly benefits the environment protection. In the paper are presented the results of tests concerning the influence of presence of fly ash (W) in CEM II and CEM IV cements produced using different method on rheological properties, air content, setting times and plastic shrinkage of mortars. Moreover compatibility of plasticizers with cements containing fly ash (W) was also studied. Additionally hydration heat of cements containing calcareous fly ash (W) were determined.

\section{Experimental}

\subsection{Research plan and variables}

Research plan is shown in Table 2. As variable factors in research were adopted:

- Cement type:

- calcareous fly ash (W) content in cement (CEM II/A-W, CEM II/B-W, CEM IV/B-W),

- presence of other mineral admixtures in cement: siliceous fly ash (V), ground granulated blast furnace slag (S), limestone (LL) (CEM II/A-M (V, W), CEM II/B-M (V, W), CEM IV/B-M (V, W), CEM II/B-M (S, W), CEM II/B-M (LL, W)).

Table 2 Experimental plan

\begin{tabular}{|c|c|c|c|}
\hline \multirow[t]{3}{*}{ Cement } & \multicolumn{3}{|c|}{ Method of cement production } \\
\hline & \multicolumn{2}{|c|}{$\begin{array}{l}\text { Blending cement CEM I with calcareous fly ash } \\
\text { W and other additives - V, S, LL }\end{array}$} & \multirow{2}{*}{$\begin{array}{c}\text { Intergriding clinker, calcareous fly ash } \mathrm{W} \text {, } \\
\text { other additives }-\mathrm{V}, \mathrm{S}, \mathrm{LL} \text { and setting regulator } \\
\text { (gypsum) }\end{array}$} \\
\hline & W unprocessed & $\mathrm{W}$ processed & \\
\hline Reference & \multicolumn{2}{|c|}{ CEM I } & CEM I (g) \\
\hline CEM II/A-W & $\mathrm{x}$ & $\mathrm{x}$ & $\mathrm{x}$ \\
\hline CEM II/B-W & $\mathrm{x}(\mathrm{PL} 1, \mathrm{PL} 2)$ & $\begin{array}{l}\mathrm{x} \text { (PL1, PL2), variability } \\
\text { of W properties }\end{array}$ & $\mathrm{x}(\mathrm{PL} 1, \mathrm{PL} 2)$ \\
\hline CEM IV/B-W & $\mathrm{x}$ & $\mathrm{x}$ & $\mathrm{x}$ \\
\hline CEM II/A-M (V, W) & $\mathrm{x}$ & $\mathrm{x}$ & $\mathrm{x}$ \\
\hline CEM II/B-M (V, W) & $\mathrm{x}$ & $\mathrm{x}(\mathrm{PL} 1, \mathrm{PL} 2)$ & $\mathrm{x}$ \\
\hline CEM IV/B $(\mathrm{V}, \mathrm{W})$ & $\mathrm{x}$ & $\mathrm{x}$ & $\mathrm{x}$ \\
\hline CEM II/B-M (S, W), (S - $15 \%, \mathrm{~W}-15 \%)$ & $\mathrm{x}$ & $\mathrm{x}(\mathrm{PL} 1, \mathrm{PL} 2)$ & $\mathrm{x}$ \\
\hline CEM II/B-M (S, W)12, (S - $10 \%, \mathrm{~W}-20 \%)$ & - & $\mathrm{x}$ & - \\
\hline CEM II/B-M (S, W)2, (S - $20 \%, \mathrm{~W}-10 \%)$ & - & $\mathrm{x}$ & - \\
\hline CEM II/B-M (LL,W) & $\mathrm{x}$ & $\mathrm{x}(\mathrm{PL} 1, \mathrm{PL} 2)$ & $\mathrm{x}$ \\
\hline
\end{tabular}


- Method of cement production:

- by intergrinding of all the constituents (clinker, fly ash (W), other non-clinker constituents (V, S, LL), gypsum in a laboratory ball-mill,

- by homogenization in blender of earlier prepared materials: Portland cement CEM I 42,5R, raw or ground fly ash (W), other non-clinker constituents (V, ground $\mathrm{S}$, ground LL).

- Processing of fly ash (W) - raw or ground fly ash (W) (in the case of cement produced by homogenization).

- Batch of fly ash (W) (CEM II/B-W obtained by homogenization using different types of fly ash W).

As reference cements CEM I were used. Cements produced by blending are marked $b u$ when raw fly ash W was used, $b p$ when processed fly ash $\mathrm{W}$ was used. Cements produced by intergrinding are marked $g$.

\subsection{Cement production method}

Homogenized cements were produced by homogenization of earlier prepared materials: Portland cement CEM I $42,5 \mathrm{R}$, raw or ground fly ash (W), other non-clinker constituents (V, ground S, ground LL) in blender ball - mill within 5 minutes.

Interground cements were produced by intergrinding of all the constituents (clinker, fly ash (W), other non-clinker constituents (V, S, LL), gypsum in a laboratory ball-mill until specific surface of $4000-4400 \mathrm{~cm}^{2} / \mathrm{g}$ was obtained. Clinker initially was milled in ball mill to the surface of the $2500 \mathrm{~cm}^{2} / \mathrm{g}$. Next clinker was ground together with gypsum to the surface of the $3600-3800 \mathrm{~cm}^{2} / \mathrm{g}$. Then fly ash (W) and other mineral admixtures were added and were ground to get established specific surface area.

\subsection{Testing methods}

Rheological properties. Influence of cements containing fly ash (W) on rheology was tested using mortars. Rheological behavior of mortar may be sufficiently described by the Bingham model according to equation:

$$
\tau=\tau_{0}+\eta_{p l} \cdot \dot{\gamma}
$$

where: $\tau(\mathrm{Pa})$ is the shear stress at shear rate $\gamma(1 / \mathrm{s})$ and $\tau_{0}$ $(\mathrm{Pa})$ and $\eta_{p l}(\mathrm{~Pa} \cdot \mathrm{s})$ are the yield stress and plastic viscosity, respectively [27, 28]. Yield stress determines the value of shear stress necessary for initiating flow. When the shear stress surpasses the yield stress, the flow of the mixture occurs and the resistance of the flow depends on plastic viscosity; the higher the plastic viscosity of the mixture, the slower it can flow. The parameter of particular importance for workability of the mixture is the yield stress $\tau_{0}$ - its value determines the occurrence of flow of the mixture, and, in consequence, the accurate performance of technological processes of concrete production. The technological meaning of the plastic viscosity $\eta_{p l}$ is marginal in normal concretes with relatively high w/c ratio. However, in the case of mixtures, with characterize by low w/c ratio and by high flow degree (low yield stress $\tau_{0}$ ) obtained thanks to addition of the superplasticizer, the plastic viscosity $\eta_{p l}$ is of significance for their workability and stability (HPC and SCC mixtures). It is necessary to notice that studies on rheology of mortars and concretes indicate that results of rheological measurements obtained for mortars may be suitable for prediction of fresh concrete rheology [28-31].

The rheological parameters of mortar or fresh concrete can be measured by applying no less than two considerably different rotation speed $N$ and the measuring the resulting torque $T$. The rheological parameters are determined by regression analysis according to the relation:

$T=g+h N$,

where $g(\mathrm{Nm})$ and $h(\mathrm{Nm}$ s) are rheological constants corresponding to yield stress $\tau_{0}$ and plastic viscosity $\eta_{p l}$, respectively [27]. After determining measurement constants of rheometer one may, if necessary, represent the values $g$ and $h$ in physical units. According to [23], in the apparatus like the one used in this work, $\tau_{0}=7.9 \mathrm{~g}$ and $\eta_{p l}=0.78 \mathrm{~h}$, but all results are given below in terms of yield stress $g$ and plastic viscosity $h$. Theoretical basis and rules for rheological measurements are discussed widely in monographic studies by [32].

The mixer and mixing procedure of mortars were compliant with [33]; plasticizer were added $30 \mathrm{sec}$. after addition of water. After mixing mortars samples were transferred to rheometer and tested. After the end of each measurement, the mortar and concrete were stored in mixer and remixed for $2 \mathrm{~min}$ before the next measurement. Simultaneously with rheological test flow test in acc. with [34] was performed.

Air content in mortar. Air content in mortars was tested in acc. with [35].The measurement was carried out after 1 minute of mixing components in a pressure apparatus with a capacity of $750 \mathrm{ml}$. The mortar was cast in two layers. Each was consolidate by a 15 -fold drop on the table from a height of $30 \mathrm{~mm}$. After dropping, the upper part of the apparatus was applied to the lower container and the measurement in a pressure apparatus was carried out. 
Setting times of mortars. The setting times for mortars were measured using the Vicat apparatus in acc. with [36]. For the determination of initial set - expressed as the elapsed time since the addition of mixing water- a round needles with a cross-sectional area $1 \mathrm{~mm}^{2}$ is used. This needle acting under the prescribed weight is used to penetrate a paste of standard consistence placed in special mold. When the morat stiffens sufficiently for the needle to penetrate only to a point $6 \mathrm{~mm}$ from the bottom initial set.

Plastic shrinkage of mortars. Plastic shrinkage was investigated using Schleibinger Shrinkage Cone by a laser measurement [37]. The tests were performed on mortars analogous to rheological tests, at a temperature of $20^{\circ} \mathrm{C}$ and a relative humidity of $60 \%$ (the apparatus was placed in a climatic chamber).

Heat hydration of cement. Heat of hydration of the cement was determined with isothermal microcalorimeters TamAir using admix ampoules. With this apparatus one determines the amount of heat in $\mathrm{J} / \mathrm{g}$ that is emitted in isothermal conditions during cement hydration from the moment of its contact with water. Measured is the heat stream that forms during the reaction of unhydrated cement sample with water and of inert referential sample of analogous heat capacity. The measurement was conducted on binder sample (CFA or CEM I cement with fly ash) weighting $5 \pm 0.001 \mathrm{~g}$, mixed with $2.5 \mathrm{~g}$ of water. The water binder ratio $(\mathrm{w} / \mathrm{b})$ of tested cement paste was 0.5 . During the measurement, temperature of the cement paste was $200^{\circ} \mathrm{C}$. Measurement of the heat of hydration had lasted 72 hours.

Compressive strength of mortars. Compressive strength of mortars was determined in acc. with [33]. For the compressive strength test, cubic specimens with dimensions of $40 \mathrm{~mm} \times 40 \mathrm{~mm} \times 160 \mathrm{~mm}$ were cast for $24 \mathrm{~h}$, followed by demolding and stored in water. Compressive strength test were conducted after aging for 28 days.

\subsection{Materials and compositions}

Calcareous fly ash (W) collected from the power plant located in Bełchatów in central Poland was used for research. Bełchatów power plant is the largest manufacturers of calcareous fly ash (W). Chemical composition, physical and chemical properties of these fly ashes (W) and their variability are shown in Table 3 .

Performed monitoring indicates the stabilization of its physical and chemical parameters in extend which is no significant object in its using in the cement production [7]. Fly ashes (W) are characterized by both pozzolanic and hydraulic activity and by more complex mineral and chemical composition than siliceous fly ash (V) [7-11]. The main
Table 3 Composition and properties of calcareous fly ash (W) Bełchatów and its variation [37]

\begin{tabular}{|c|c|c|}
\hline constituent/property & Average & Variation \\
\hline LOI, \% & 3.7 & $1.6-7.2$ \\
\hline $\mathrm{SiO}_{2}, \%$ & 43 & $29-56$ \\
\hline $\mathrm{SiO}_{2}$ reactive, $\%$ & 24 & $25-35$ \\
\hline $\mathrm{Al}_{2} \mathrm{O}_{3}, \%$ & 19 & $10-27$ \\
\hline $\mathrm{Fe}_{2} \mathrm{O}_{3}, \%$ & 4.5 & $2.4-7.2$ \\
\hline $\mathrm{CaO}, \%$ & 24 & $13-27$ \\
\hline $\mathrm{CaO}$ reactive, $\%$ & 21 & $18-28$ \\
\hline $\mathrm{MgO}, \%$ & 1.4 & $0.9-1.9$ \\
\hline $\mathrm{SO}_{3}, \%$ & 2.7 & $0.6-6.1$ \\
\hline $\mathrm{K}_{2} \mathrm{O}, \%$ & 0.2 & $0.1-0.7$ \\
\hline $\mathrm{Na}_{2} \mathrm{O}, \%$ & 0.2 & $0.04-0.37$ \\
\hline $\mathrm{TiO}_{2}, \%$ & 1.3 & $0.8-2.3$ \\
\hline $\mathrm{CaO}_{\text {free }}, \%$ & 2.1 & $0.2-5.9$ \\
\hline Volume density, $\mathrm{kg} / \mathrm{m}^{3}$ & 920 & $850-1100$ \\
\hline Fineness, $\%$ of mass of grains $>0,045 \mathrm{~mm}$ & 51 & $35-65$ \\
\hline Water demandness, $\%$ & 113 & $102-120$ \\
\hline
\end{tabular}

mineral components are as follows: quartz, gehlenite, anorthite, anhydrite and calcium oxide, typical cement clinker phases, i.e. $\mathrm{C}_{2} \mathrm{~S}, \mathrm{C}_{12} \mathrm{~A}_{7}, \mathrm{C}_{4} \mathrm{AF}, \mathrm{C}_{4} \mathrm{~A}_{3} \mathrm{~S}$ are also identified - these phases determine hydraulic properties of fly ashes (W) [7-11]. The pozzolanic activity is determined by the presence of reactive silicon dioxide $\left(\mathrm{SiO}_{2}\right)$ and alumina $\left(\mathrm{Al}_{2} \mathrm{O}_{3}\right)$ [7-11]. Pozzolanic and hydraulic properties of fly ashes (W) are also related to amorphous phase content calcium aluminosilicate glass [7-11]. Contents of unburned carbon in being discussed fly ashes (W) do not exceed $4.5 \%$ and on average is $2.7 \%$. Participation of reactive calcium oxide is well above $10 \%$, and the content of reactive silicon dioxide over $25 \%$. Characteristics of chemical composition and phase allows to classify calcareous fly ash (W) as calcium aluminosilicate [7-11]. Researches and analyses concerning the use of fly ashes (W) proved that quality requirements of standard [1] are met and it is possible to use them as a main cement constituent $[7,8,10]$.

For the production of homogenized and interground cements cement CEM I $42.5 \mathrm{R}$ and clinker was used respectively of properties presented in Table 4 .

Properties of calcareous fly ash (W), siliceous fly ash (V), ground granulated blast furnace slag (S) and ground limestone (LL) used for cement production are presented in Table 4. Influence of calcareous fly ash (W) properties on CEM II/B-W cement was investigated using six fly ashes of properties presented in Table 5 .

The fly ashes $\mathrm{W}$ were taken from the technological batches through the course of three months. Composition and properties of cement used are presented in Tables 6-8. 
Table 4 Properties of cements constituents

\begin{tabular}{|c|c|c|c|c|c|c|c|c|c|c|c|}
\hline \multirow{2}{*}{$\begin{array}{l}\text { Cement for blended cements and } \\
\text { cements constituents type }\end{array}$} & \multicolumn{9}{|c|}{ Ingredient, $\%$ of mass } & \multirow{2}{*}{$\begin{array}{c}\text { Density, } \\
\mathrm{g} / \mathrm{cm}^{3}\end{array}$} & \multirow{2}{*}{$\begin{array}{l}\text { Blain specific } \\
\text { surface, } \mathrm{cm}^{2} / \mathrm{g}\end{array}$} \\
\hline & LOI & $\mathrm{SiO}_{2}$ & $\mathrm{Al}_{2} \mathrm{O}_{3}$ & $\mathrm{Fe}_{2} \mathrm{O}_{3}$ & $\mathrm{CaO}$ & $\mathrm{MgO}$ & $\mathrm{SO}_{3}$ & $\mathrm{~K}_{2} \mathrm{O}$ & $\mathrm{Na}_{2} \mathrm{O}$ & & \\
\hline CEM I for blended cements & - & 19 & 4.9 & 2.9 & 63 & 1.3 & 2.8 & 0.9 & 0.14 & 3.09 & 3630 \\
\hline Clinker for interground cements & 1.9 & 20 & 4.5 & 2.1 & 67 & 1.0 & - & 0.24 & 0.54 & - & - \\
\hline $\begin{array}{l}\text { Calcareous fly } \\
\text { ash } W^{1}\end{array}$ & 2.6 & 34 & 19 & 5.4 & 31 & 1.8 & 4.3 & 0.11 & 0.31 & - & $\begin{array}{l}2860 \\
3500\end{array}$ \\
\hline Siliceous fly ash V & 2.3 & 54 & 27 & 5.8 & 3 & 2.7 & 0.2 & 3.31 & 0.84 & 2.24 & 4100 \\
\hline Slag S & 1.1 & 37 & 7 & 1.2 & 46 & 5.2 & 2.1 & 0.39 & 0.53 & 2.75 & 2520 \\
\hline Limestone LL & 44 & 1.3 & 0.6 & 0.2 & 54 & 0.5 & 0.03 & 0.03 & 0.01 & 2.93 & 4020 \\
\hline
\end{tabular}

${ }^{1}$ sieve residue $(0.045 \mathrm{~mm})$ - raw calcareous fly ash $\mathrm{W}-36.4 \%$, processed calcareous fly ash $\mathrm{W}-23 \%$,

Table 5 Properties of calcareous fly ash W used in research on influence of fly ash type on rheological properties of CEM II/B-W cements

\begin{tabular}{|c|c|c|c|c|c|c|c|c|c|c|c|c|c|}
\hline \multirow{2}{*}{$\begin{array}{l}\text { CFA Type of } \\
\text { CEM II/B-W }\end{array}$} & \multicolumn{10}{|c|}{ Ingredient, $\%$ of mass } & \multicolumn{2}{|c|}{ Fineness, $\%$} & \multirow{2}{*}{$\begin{array}{c}\text { Volume } \\
\text { density, } \mathrm{kg} / \mathrm{m}^{3}\end{array}$} \\
\hline & LOI & $\mathrm{SiO}_{2}$ & $\mathrm{Al}_{2} \mathrm{O}_{3}$ & $\mathrm{Fe}_{2} \mathrm{O}_{3}$ & $\mathrm{CaO}$ & $\mathrm{MgO}$ & $\mathrm{SO}_{3}$ & $\mathrm{~K}_{2} \mathrm{O}$ & $\mathrm{Na}_{2} \mathrm{O}$ & $\mathrm{CaO}_{\mathrm{w}}$ & raw & processed & \\
\hline A & 2.6 & 33.5 & 19.2 & 5.4 & 31.2 & 1.84 & 4.33 & 0.11 & 0.31 & 3.43 & 36.4 & 23 & 1098 \\
\hline B & 3.4 & 35.4 & 21.9 & 6.1 & 25.6 & 1.49 & 4.22 & 0.13 & 0.16 & 1.24 & 35.4 & 13.3 & 749 \\
\hline $\mathrm{C}$ & 1.8 & 40.2 & 24.0 & 5.9 & 22.4 & 1.27 & 2.49 & 0.2 & 0.15 & 1.46 & 55.6 & 22 & 1059 \\
\hline $\mathrm{D}$ & 2.2 & 41.0 & 18.5 & 5.0 & 25.4 & 1.43 & 4.25 & 0.18 & 0.18 & 1.92 & 50.2 & 22 & 845 \\
\hline E & 3.0 & 41.0 & 15.1 & 3.6 & 30.1 & 1.57 & 3.27 & 0.21 & 0.31 & 4.57 & 47.4 & 21 & 934 \\
\hline $\mathrm{F}$ & 3.6 & 56.9 & 18.2 & 3.2 & 14.1 & 0.94 & 1.6 & 0.14 & 0.11 & 1.71 & 60.4 & 25 & 1058 \\
\hline
\end{tabular}

Table 6 Chemical properties of various types of cements

\begin{tabular}{|c|c|c|c|c|c|c|c|c|}
\hline \multirow{2}{*}{ Cement type } & \multicolumn{8}{|c|}{ Ingredient, $\%$ of mass } \\
\hline & LOI & $\mathrm{SiO}_{2}$ & $\mathrm{Al}_{2} \mathrm{O}_{3}$ & $\mathrm{Fe}_{2} \mathrm{O}_{3}$ & $\mathrm{CaO}$ & $\mathrm{K}_{2} \mathrm{O}$ & $\mathrm{Na}_{2} \mathrm{O}$ & $\mathrm{SO}_{3}$ \\
\hline CEM I & 2.65 & 19.18 & 4.93 & 2.70 & 65.08 & 0.79 & 0.12 & 2.74 \\
\hline CEM I (g) & 1.92 & 20.35 & 4.48 & 2.06 & 66.56 & 0.54 & 0.24 & 2.84 \\
\hline \multicolumn{9}{|c|}{ CEM II/A- W, CEM II/B-W, CEM IV/B-W } \\
\hline CEM II/A-W bu & 3.31 & 21.13 & 7.02 & 3.03 & 59.39 & 0.72 & 0.15 & 3.17 \\
\hline CEM II/B-W bu & 3.24 & 22.85 & 9.07 & 3.46 & 54.80 & 0.62 & 0.18 & 3.45 \\
\hline CEM IV/B-W bu & 3.27 & 25.86 & 12.23 & 4.00 & 47.39 & 0.47 & 0.22 & 3.73 \\
\hline CEM II/A-W bp & 3.88 & 20.33 & 6.87 & 3.10 & 59.96 & 0.69 & 0.15 & 3.1 \\
\hline CEM II/B-W bp & 3.58 & 23.03 & 9.16 & 3.48 & 54.39 & 0.58 & 0.18 & 3.53 \\
\hline CEM IV/B-W bp & 3.36 & 25.34 & 11.95 & 4.07 & 48.15 & 0.46 & 0.22 & 3.84 \\
\hline CEM II/A-W g & 2.01 & 22.38 & 6.60 & 2.54 & 61.29 & 0.16 & 0.25 & 2.84 \\
\hline CEM II/B-W g & 2.19 & 23.89 & 8.71 & 3.07 & 56.56 & 0.15 & 0.26 & 3.05 \\
\hline CEM IV/B-W g & 2.30 & 25.97 & 11.54 & 3.76 & 50.28 & 0.15 & 0.28 & 3.18 \\
\hline \multicolumn{9}{|c|}{ CEM II/A-M (V, W), CEM II/B-M (V, W), CEM IV/B (V, W) } \\
\hline CEM II/A-M $(\mathrm{V}, \mathrm{W})$ bu & 3.49 & 22.42 & 7.47 & 3.20 & 57.26 & 0.96 & 0.19 & 3.06 \\
\hline CEM II/B-M $(\mathrm{V}, \mathrm{W})$ bu & 3.44 & 26.42 & 10.34 & 3.65 & 49.42 & 1.10 & 0.27 & 2.77 \\
\hline CEM IV/B $(\mathrm{V}, \mathrm{W})$ bu & 3.14 & 30.40 & 13.70 & 4.44 & 40.90 & 1.27 & 0.36 & 2.74 \\
\hline CEM II/A-M $(V, W) b p$ & 3.87 & 21.59 & 7.83 & 3.24 & 57.40 & 1.03 & 0.22 & 2.88 \\
\hline CEM II/B-M (V, W) bp & 3.87 & 25.11 & 10.16 & 3.51 & 50.90 & 1.06 & 0.26 & 2.84 \\
\hline CEM IV/B $(V, W)$ bp & 3.60 & 30.28 & 14.44 & 4.09 & 40.29 & 1.25 & 0.37 & 2.84 \\
\hline CEM II/A-M $(\mathrm{V}, \mathrm{W}) \mathrm{g}$ & 1.88 & 23.97 & 7.23 & 2.76 & 58.60 & 0.40 & 0.29 & 2.82 \\
\hline CEM II/B-M (V, W) g & 2.05 & 26.47 & 9.52 & 3.28 & 52.42 & 0.61 & 0.34 & 3.16 \\
\hline CEM IV/B $(\mathrm{V}, \mathrm{W}) \mathrm{g}$ & 2.74 & 28.82 & 10.14 & 3.04 & 48.28 & 0.93 & 0.36 & 3.19 \\
\hline
\end{tabular}


Continuation of Table 6

\begin{tabular}{|c|c|c|c|c|c|c|c|c|}
\hline \multirow{2}{*}{ Cement type } & \multicolumn{8}{|c|}{ Ingredient, $\%$ of mass } \\
\hline & LOI & $\mathrm{SiO}_{2}$ & $\mathrm{Al}_{2} \mathrm{O}_{3}$ & $\mathrm{Fe}_{2} \mathrm{O}_{3}$ & $\mathrm{CaO}$ & $\mathrm{K}_{2} \mathrm{O}$ & $\mathrm{Na}_{2} \mathrm{O}$ & $\mathrm{SO}_{3}$ \\
\hline \multicolumn{9}{|c|}{ CEM II/B-M (S, W) } \\
\hline CEM II/B-M (S, W) bu & 2.56 & 23.87 & 7.37 & 2.93 & 56.81 & 0.64 & 0.21 & 3.17 \\
\hline CEM II/B-M (S, W) bp & 2.51 & 23.87 & 7.37 & 2.92 & 56.87 & 0.63 & 0.22 & 3.19 \\
\hline CEM II/B-M (S, W)21 bp & 2.50 & 23.90 & 7.97 & 3.02 & 56.25 & 0.61 & 0.20 & 3.22 \\
\hline CEM II/B-M (S, W)12 bp & 2.34 & 24.57 & 6.83 & 2.57 & 57.31 & 0.66 & 0.23 & 2.98 \\
\hline CEM II/B-M (S, W) g & 1.92 & 24.49 & 6.99 & 2.44 & 57.91 & 0.19 & 0.30 & 3.34 \\
\hline \multicolumn{9}{|c|}{ CEM II/B-M (LL, W) } \\
\hline CEM II/B-M (LL, W) bu & 6.79 & 18.65 & 6.42 & 2.76 & 60.05 & 0.58 & 0.14 & 3.17 \\
\hline CEM II/B-M (LL, W) bp & 6.59 & 18.55 & 6.40 & 2.80 & 60.33 & 0.59 & 0.14 & 3.19 \\
\hline CEM II/B-M (LL, W) g & 6.10 & 19.60 & 6.15 & 2.30 & 60.68 & 0.14 & 0.22 & 3.22 \\
\hline
\end{tabular}

Table 7 Physical properties of various types of cements

\begin{tabular}{|c|c|c|c|c|c|c|c|c|c|}
\hline \multirow{2}{*}{ Cement type } & \multicolumn{5}{|c|}{ Constituents, $\%$} & \multirow{2}{*}{$\begin{array}{l}\text { Density, } \\
\mathrm{g} / \mathrm{cm}^{3}\end{array}$} & \multirow{2}{*}{$\begin{array}{c}\text { Water } \\
\text { demand, \% }\end{array}$} & \multirow{2}{*}{$\begin{array}{l}\text { Blain specific } \\
\text { surface, } \mathrm{cm}^{2} / \mathrm{g}\end{array}$} & \multirow{2}{*}{$\begin{array}{c}\text { Compressive } \\
\text { strength } \\
28 \text { days, } \mathrm{MPa}\end{array}$} \\
\hline & CEM I & Clinker & $\mathrm{W}$ & $\mathrm{V} / \mathrm{S} / \mathrm{LL}$ & Gypsum & & & & \\
\hline CEM I & 100 & & & & & 3.09 & 27.6 & 3630 & 50.2 \\
\hline CEM I (g) & & 95 & - & - & 5 & 3.10 & 25.8 & 3810 & 59.2 \\
\hline \multicolumn{10}{|c|}{ CEM II/A- W, CEM II/B-W, CEM IV/B-W } \\
\hline CEM II/A-W bu & 85 & - & 15 & - & - & 2.99 & 3640 & 2650 & 53.2 \\
\hline CEM II/B-W bu & 70 & - & 30 & - & - & 2.95 & 3570 & 2800 & 49.6 \\
\hline CEM IV/B-W bu & 50 & - & 50 & - & - & 2.85 & 3420 & 3020 & 38.8 \\
\hline CEM II/A-W bp & 85 & - & 15 & - & - & 3.04 & 4020 & 3000 & 56.5 \\
\hline CEM II/B-W bp & 70 & - & 30 & - & - & 2.99 & 4070 & 3100 & 53.4 \\
\hline CEM IV/B-W bp & 50 & - & 50 & - & - & 2.92 & 4150 & 3220 & 49.2 \\
\hline CEM II/A-W g & - & 81.1 & 14.3 & - & 4.6 & 3.04 & 4190 & 2760 & 58.7 \\
\hline CEM II/B-W g & - & 67.7 & 29 & - & 3.3 & 2.98 & 4030 & 3040 & 51.1 \\
\hline CEM IV/B-W g & - & 49.2 & 49.2 & - & 1.6 & 2.88 & 4000 & 3140 & 42.1 \\
\hline \multicolumn{10}{|c|}{ CEM II/A-M (V, W), CEM II/B-M (V, W), CEM IV/B (V, W) } \\
\hline CEM II/A-M (V, W) bu & 85 & - & 7.5 & 7.5 & - & 3.06 & 26.8 & 3960 & 51.6 \\
\hline CEM II/B-M $(\mathrm{V}, \mathrm{W})$ bu & 70 & - & 15 & 15 & - & 2.77 & 27.4 & 3840 & 47.6 \\
\hline CEM IV/B $(\mathrm{V}, \mathrm{W})$ bu & 50 & - & 25 & 25 & - & 2.74 & 28.6 & 3700 & 34.6 \\
\hline CEM II/A-M $(\mathrm{V}, \mathrm{W}) \mathrm{bp}$ & 85 & - & 7.5 & 7.5 & - & 2.88 & 25.9 & 3880 & 53.1 \\
\hline CEM II/B-M (V, W) bp & 70 & - & 15 & 15 & - & 2.84 & 26.2 & 3820 & 50.9 \\
\hline CEM IV/B $(\mathrm{V}, \mathrm{W}) \mathrm{bp}$ & 50 & - & 25 & 25 & - & 2.84 & 26.9 & 3820 & 37.7 \\
\hline CEM II/A-M $(\mathrm{V}, \mathrm{W}) \mathrm{g}$ & - & 80.5 & 7.1 & 7.1 & 5.3 & 2.82 & 27.4 & 3970 & 54.9 \\
\hline CEM II/B-M $(\mathrm{V}, \mathrm{W}) \mathrm{g}$ & - & 66.7 & 14.3 & 14.3 & 4.7 & 3.16 & 28.6 & 4130 & 47.7 \\
\hline CEM IV/B $(\mathrm{V}, \mathrm{W}) \mathrm{g}$ & - & 48.1 & 24 & 24 & 3.9 & 3.19 & 29.6 & 4130 & 36.4 \\
\hline \multicolumn{10}{|c|}{ CEM II/B-M (S, W) } \\
\hline CEM II/B-M (S, W) bu & 70 & - & 15 & 15 & - & 2.99 & 30.8 & 3810 & 56.7 \\
\hline CEM II/B-M (S, W) bp & 70 & - & 15 & 15 & - & 3.01 & 31.2 & 4060 & 56.9 \\
\hline CEM II/B-M (S, W)21 bp & - & 70 & 20 & 10 & - & 2.98 & 26.7 & 3680 & 54.7 \\
\hline CEM II/B-M (S, W)12 bp & - & 70 & 10 & 20 & - & 2.98 & 27.2 & 3610 & 54.5 \\
\hline CEM II/B-M (S, W) g & - & 64.7 & 15.3 & 15.3 & 4.7 & 3.00 & 28.2 & 4060 & 56.6 \\
\hline \multicolumn{10}{|c|}{ CEM II/B-M (LL, W) } \\
\hline CEM II/B-M (LL, W) bu & 70 & - & 15 & 15 & - & 2.96 & 30.4 & 4230 & 45.1 \\
\hline CEM II/B-M (LL, W) bp & 70 & - & 15 & 15 & - & 2.98 & 30.6 & 4340 & 46.0 \\
\hline CEM II/B-M (LL, W) g & - & 64.7 & 15.3 & 15.3 & 4.7 & 2.97 & 27.2 & 4430 & 47.4 \\
\hline
\end{tabular}


Table 8 Properties of cements CEM II/B-W for investigation influence of batch of fly ash (W) on rheological properties and setting time of mortars

\begin{tabular}{|c|c|c|c|c|c|c|c|}
\hline \multirow{2}{*}{ Cement } & \multirow{2}{*}{$\begin{array}{l}\text { Density } \\
\mathrm{g} / \mathrm{cm}^{3}\end{array}$} & \multirow{2}{*}{$\begin{array}{l}\text { Blain specific } \\
\text { surface, } \mathrm{cm}^{2} / \mathrm{g}\end{array}$} & \multirow{2}{*}{$\begin{array}{c}\text { Water } \\
\text { demand, \% }\end{array}$} & \multicolumn{2}{|c|}{ Flow, cm } & \multicolumn{2}{|c|}{ Setting time, $\min$} \\
\hline & & & & po $5 \mathrm{~min}$ & po $90 \mathrm{~min}$ & Initial & End \\
\hline CEM II/B-W A bu & 2.95 & 3570 & 28.0 & 20.8 & 17.8 & 188 & 298 \\
\hline CEM II/B-W B bu & 2.93 & 3930 & 35.6 & 18.0 & - & 214 & 354 \\
\hline CEM II/B-W C bu & 2.93 & 3630 & 29.8 & 20.6 & 18.2 & 198 & 348 \\
\hline CEM II/B-W D bu & 2.95 & 3580 & 30.6 & 19.5 & - & 221 & 321 \\
\hline CEM II/B-W E bu & 2.96 & 3530 & 31.0 & 20.3 & 18.2 & 197 & 347 \\
\hline CEM II/B-W F bu & 2.95 & 3180 & 30.0 & 20.1 & 17.9 & 215 & 375 \\
\hline CEM II/B-W A bp & 2.99 & 4070 & 31.0 & 21.3 & 19.5 & 186 & 296 \\
\hline CEM II/B-W B bp & 2.95 & 4440 & 34.2 & 18.1 & - & 229 & 309 \\
\hline CEM II/B-W C bp & 2.96 & 4030 & 29.8 & 22.0 & 20.0 & 222 & 322 \\
\hline CEM II/B-W D bp & 2.99 & 4300 & 30.8 & 20.0 & 17.0 & 201 & 291 \\
\hline CEM II/B-W E bp & 2.98 & 4230 & 30.8 & 22.0 & 19.7 & 188 & 318 \\
\hline CEM II/B-W F bp & 2.98 & 3930 & 29.8 & 22.5 & 19.4 & 222 & 235 \\
\hline
\end{tabular}

Properties of plasticizers are shown in Table 9. Two representative plasticizers from among those commercially available were selected. Plasticisers differ in their chemical bases.

Proportions of mortars used for testing rheological properties and plastic shrinkage are shown in Table 10. Standard sand in acc. with [33] was used. The proportions of mortars mixture were based on standard mortar proportioning according to [33] but with w/c ratio changed to 0.55 . In the other tests proportioning of mortars was in acc. with requirements of adequate standards.

\section{Test results and discussion}

Influence of cements containing calcareous fly ash (W) on rheological properties, air content, setting times and plastic shrinkage of mortars are presented on Figs. 1-11 and in Table 11. Heat of hydration of these cements is presented in Table 11.

Table 9 Properties of plasticizers

\begin{tabular}{lcc}
\hline Symbol & Chemical base & Dosage \\
\hline PL1 & lignosulfonate & $1 / 2 \max =0.25 \%$ \\
& $\begin{array}{c}\text { Iminodiethanol, bis ethanol, phosphate } \\
\text { PL2 }\end{array}$ & $\begin{array}{c}\text { (V) tri butyl acetate, formaldehyde, } \\
\text { methanol, (Z)-octadec-9-enyloamine }\end{array}$ \\
& & $1 / 2 \max =0.25 \%$ \\
\hline
\end{tabular}

Table 10 Mortar proportioning

\begin{tabular}{lc}
\hline & Content, g/batch \\
\hline Cement & 450 \\
Sand & 1350 \\
Water & 247.5 \\
w/c & 0.55 \\
\hline
\end{tabular}

\subsection{Cements CEM II/A-W, CEM II/B-W, CEM IV/B-W}

Regardless of the production method of cement, CEM II/ A-Wmortars are characterized by higher yield stress $g$ (and smaller flow diameter) than CEM I mortars, and faster increase of the yield stress $g$ in time (reduction of flow diameter in time). With the increase of the amount of fly ash (W) in the cement (CEM II/B-W, CEM IV/B-W), the yield stress $g$ of mortars and its increase in time increase. The negative influence of the presence of fly ash (W) in cement on the rheological properties of mortars is the strongest in case of cements blended with raw fly ash (W), and the weakest in case of interground cements - this is due to the beneficial effect of using processed fly ash. In case of mortars with blended cements with processed fly ash and with interground cements with the amount of fly ash (W) up to $30 \%$ (CEM II/A-W and CEM II/B-W) the worsening of fluidity is moderate, and becomes significantly higher only when the amount of fly ash $(\mathrm{W})$ is increased to $50 \%(\mathrm{CEM} I \mathrm{~V} / \mathrm{B}-\mathrm{W})$. In case of using cements with raw fly ash (W) the significant worsening of fluidity can be observed already when the amount of fly ash reaches $15 \%$ (CEM II/A-W). The mortars with calcareous fly ash (W) are usually characterized by the higher plastic viscosity $h$ than the mortars with cement CEM I, and with the increase of fly ash (W) content, the plastic viscosity $h$ also increases (Fig. 1).

The nature of the influence of fly ash (W) on the rheological properties of mortars when it is used as a cement constituent or as a type II additive is analogous (Fig. 2).

However, if fly ash (W) is used as the cement constituent, its negative influence on the rheological properties is clearly lower. It is probably caused by the additional 
Table 11 Influence of various cements type on properties of mortars

\begin{tabular}{|c|c|c|c|c|c|c|c|c|}
\hline \multirow{2}{*}{ Cement type } & \multicolumn{2}{|c|}{ Flow. mm } & \multirow[t]{2}{*}{ Air content. \% } & \multicolumn{2}{|c|}{ Setting time. min } & \multicolumn{3}{|c|}{ Hydration heat. J/g } \\
\hline & $5 \mathrm{~min}$ & $90 \mathrm{~min}$ & & initial & end & $12 \mathrm{~h}$ & $24 \mathrm{~h}$ & $72 \mathrm{~h}$ \\
\hline CEM I & 21.5 & 19.7 & 5.4 & 121 & 215 & 59.76 & 146.1 & 250.0 \\
\hline CEM I (g) & 20.8 & 17.8 & 2.5 & 135 & 180 & 66.96 & 161.1 & 266.6 \\
\hline \multicolumn{9}{|c|}{ CEM II/A-W, CEM II/B-W, CEM IV/B-W } \\
\hline CEM II/A-W bu & 21.0 & 17.0 & 4.2 & 135 & 180 & 56.41 & 143.0 & 243.9 \\
\hline CEM II/B-W bu & 20.4 & 17.3 & 3.4 & 188 & 298 & 45.21 & 117.7 & 226.5 \\
\hline CEM IV/B-W bu & 19.6 & 16.5 & 4 & 193 & 348 & 39.04 & 95.62 & 208.2 \\
\hline CEM II/A-W bp & 21.0 & 19.0 & 1.6 & 136 & 211 & 57.53 & 152.8 & 244.5 \\
\hline CEM II/B-W bp & 21.2 & 19.3 & 2.5 & 186 & 296 & 45.45 & 124.0 & 221.7 \\
\hline CEM IV/B-W bp & 20.5 & 16.7 & 3.4 & 276 & 346 & 45.09 & 121.9 & 210.7 \\
\hline CEM II/A-W g & 20.4 & 16.9 & 2.4 & 153 & 223 & 63.12 & 156.7 & 261.4 \\
\hline CEM II/B-W g & 20.7 & 17.0 & 2.6 & 180 & 250 & 50.47 & 149.7 & 241.2 \\
\hline CEM IV/B-W g & 19.3 & 16.8 & 2.9 & 188 & 258 & 40.45 & 109.5 & 205.9 \\
\hline \multicolumn{9}{|c|}{ CEM II/A-M (V, W),CEM II/B-M (V, W), CEM IV/B (V, W) } \\
\hline CEM II/A-M (V, W) bu & 22.1 & 19.5 & 2.2 & 171 & 241 & 42.48 & 120.4 & 221.2 \\
\hline CEM II/B-M (V, W) bu & 22.6 & 20.0 & 2.1 & 258 & 428 & 32.34 & 99.57 & 198.4 \\
\hline CEM IV/B $(V, W)$ bu & 23.0 & 19.4 & 2.4 & 202 & 442 & 26.79 & 80.70 & 175.7 \\
\hline CEM II/A-M (V, W) bp & 22.7 & 20.5 & 2.2 & 166 & 291 & 41.68 & 127.0 & 223.1 \\
\hline CEM II/B-M (V, W) bp & 23.2 & 21.1 & 2.5 & 184 & 359 & 39.28 & 117.9 & 206.9 \\
\hline CEM IV/B (V, W) bp & 22.9 & 21.5 & 2 & 274 & 449 & 26.67 & 84.30 & 166.8 \\
\hline CEM II/A-M $(\mathrm{V}, \mathrm{W}) \mathrm{g}$ & 21.4 & 19.6 & 2 & 135 & 180 & 43.37 & 118.4 & 255.3 \\
\hline CEM II/B-M (V, W) g & 21.8 & 20.3 & 2.2 & 156 & 268 & 34.44 & 106.7 & 236.8 \\
\hline CEM IV/B $(\mathrm{V}, \mathrm{W}) \mathrm{g}$ & 22.3 & 19.9 & 2.2 & 192 & 298 & 27.52 & 89.07 & 207.2 \\
\hline \multicolumn{9}{|c|}{ CEM II/B-M (S, W) } \\
\hline CEM II/B-M (S, W) bu & 22.3 & 20.0 & 1.8 & 168 & 306 & 50.32 & 130.6 & 210.5 \\
\hline CEM II/B-M (S, W) bp & 21.8 & 18.7 & 2.3 & 181 & 296 & 53.43 & 133.5 & 215.2 \\
\hline CEM II/B-M (S, W)21 bp & 21.9 & 19.0 & 2.6 & 210 & 320 & 54.94 & 134.0 & 217.1 \\
\hline CEM II/B-M (S, W)12 bp & 21.7 & 18.8 & 2.5 & 195 & 285 & 55.73 & 137.8 & 220.9 \\
\hline CEM II/B-M (S, W) g & 19.5 & 16.7 & 2.3 & 170 & 290 & 50.55 & 124.4 & 251.8 \\
\hline \multicolumn{9}{|c|}{ CEM II/B-M (LL, W) } \\
\hline CEM II/B-M (LL, W) bu & 20.7 & 17.8 & 2.2 & 175 & 250 & 57.40 & 136.3 & 214.4 \\
\hline CEM II/B-M (LL, W) bp & 21.5 & 17.5 & 2.3 & 174 & 254 & 57.23 & 136.0 & 213.9 \\
\hline CEM II/B-M (LL, W) g & 19.0 & 16.8 & 2.5 & 150 & 370 & 56.02 & 130.9 & 245.2 \\
\hline
\end{tabular}

grinding of the fly ash (W) during the process of cement production. Using the fly ash (W) as a cement constituent enables to obtain the mortars (and concrete mixes) with better workability when analogous amount of fly ash (W) is added directly to the mix.

The presence of fly ash (W) in cement does not affect or decreases of the amount of air in the mortar in comparison to mortars with CEM I cement (Table 11). Cements with fly ash (W) are characterized by the delayed initial and final setting times in relation to CEM I cement (Table 11). The greater the delay, the higher the fly ash (W) content. The delay of the initial setting time depends to a small degree on the method of cement production, whereas the delay of final setting time is the highest in case of the blended cements with processed fly ash (W), and the lowest for interground cements. Plastic shrinkage of the mortars with fly ash (W) cements is higher than of CEM I mortars and it raises proportionally to the final setting time of cement.

In the time range of first 12 to 72 hours, the amount of heat generated by cements with fly ash is smaller than by CEM I cements - in case of CEM II/A-W by about $3 \%$, CEM II/B-W by approx. $10 \%$, CEM IV/B-W by approx. $20 \%$ (Table 11). The method of cement production does not affect the amount of heat generated during the hydration. 

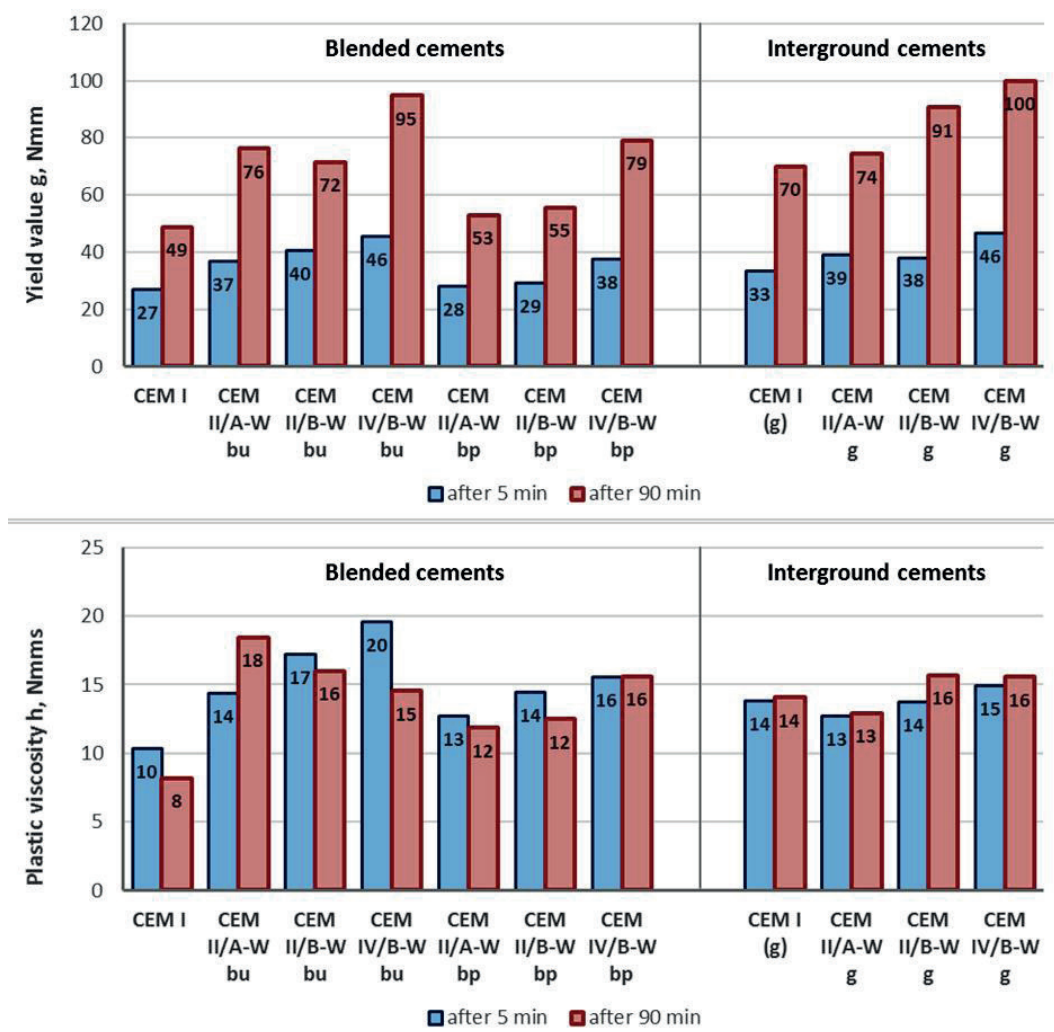

Fig. 1 Rheological properties of mortars made of cements CEM II/A-W, CEM II/B-W and CEM IV/B-W, $b u$ - cements produced by blending with raw fly ash $\mathrm{W}, b p$ - cements produced by blending with processed fly ash $\mathrm{W}, g$ - cements produced by intergrinding

\subsection{Influence of the batch of calcareous fly ash on the rheological properties of mortars with cement CEM II/B-W}

The results of the rheological tests of mortars with CEM II/B-W obtained by mixing cement CEM I with raw or processed by grinding calcareous fly ashes from different batches, are shown in Fig. 3.

Obtained results confirm a significant influence of the batch of fly ash W (its physicochemical properties) on the rheological properties of cement CEM II/B-W. They also confirm that processing of calcareous fly ash $\mathrm{W}$ by grinding reduces its impact on the rheological properties of mortars. The variation of the yield stress $g$ of mortars with cements CEM II/B-W both with raw and the processed fly ash $\mathrm{W}$ is high, and significantly higher than for other types of cement (Fig. 4).

Analysis of the obtained results indicates, that significant worsening of the rheological properties of mortars occurs when the fly ashes $\mathrm{W}$ used for cement production have a bulk density of less than $900 \mathrm{~kg} / \mathrm{m}^{3}$. It is worthy to note, that bulk density is closely related to the conditions of combustion of coal or more accurately to temperature - the lower temperature, the lower amount of the glass phase and the greater the bulk density of the fly ash W.
As consequence of lower amount of the glass phase is the higher water demand of fly ash W. It should be also noted that in case of fly ashes with bulk density of less than 900 $\mathrm{kg} / \mathrm{m}^{3}$, processing by grinding improves the rheological properties of mortars only to a small degree. If such batches of calcareous fly ashes $\mathrm{W}$ are not used for the production of cement, it is possible to achieve significant improvement in the uniformity of the rheological properties of mortars, to a level not deviating significantly from other types of cement with additives (Fig. 5). It should be noted, however, that the mortars with cement CEM II/B-W are characterized by higher workability loss (increase of yield stress $g$ in time). Yield value $g$ of cement CEM II/B-W mortars vs. volume density of fly ash $\mathrm{W}$ describes in Fig. 6.

\subsection{Cements CEM II/A-M (V-W), CEM II/B- M (V-W), CEM IV/B- M (V-W)}

Mortars with CEM II/A-M (V, W), CEM II/B-M (V, W) and CEM IV/B-M (V, W) cements are characterized by significantly lower yield stress $g$ (higher flow diameter) than the mortars with analogical cements containing only flay ash W, even lower than mortars with cement CEM I. In the same time mortars these mortars are characterized by similar or slightly lower plastic viscosity $h$ than the mortars with 

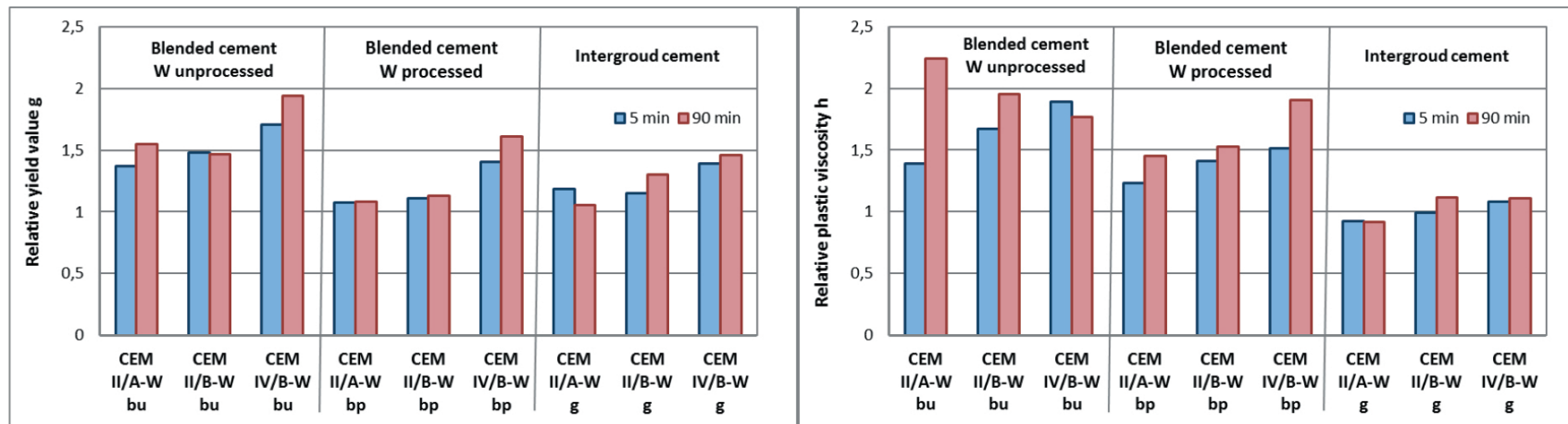

CEM II/A-W, CEM II/B-W, CEM IV/B-W
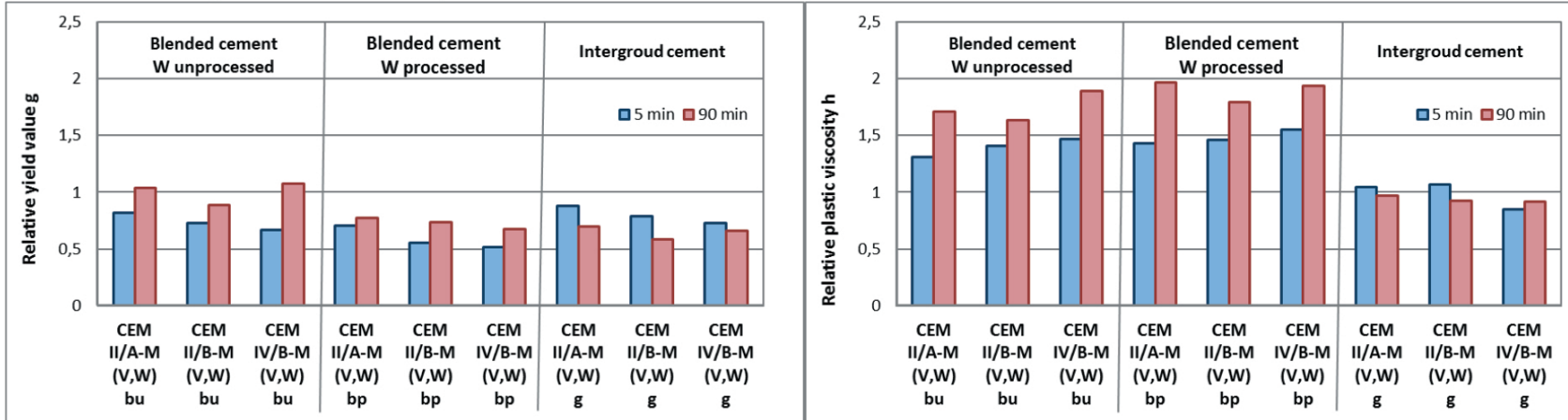

\section{CEM II/A-M (V,W), CEM II/B-M (V,W), CEM IV/B-M (V,W)}
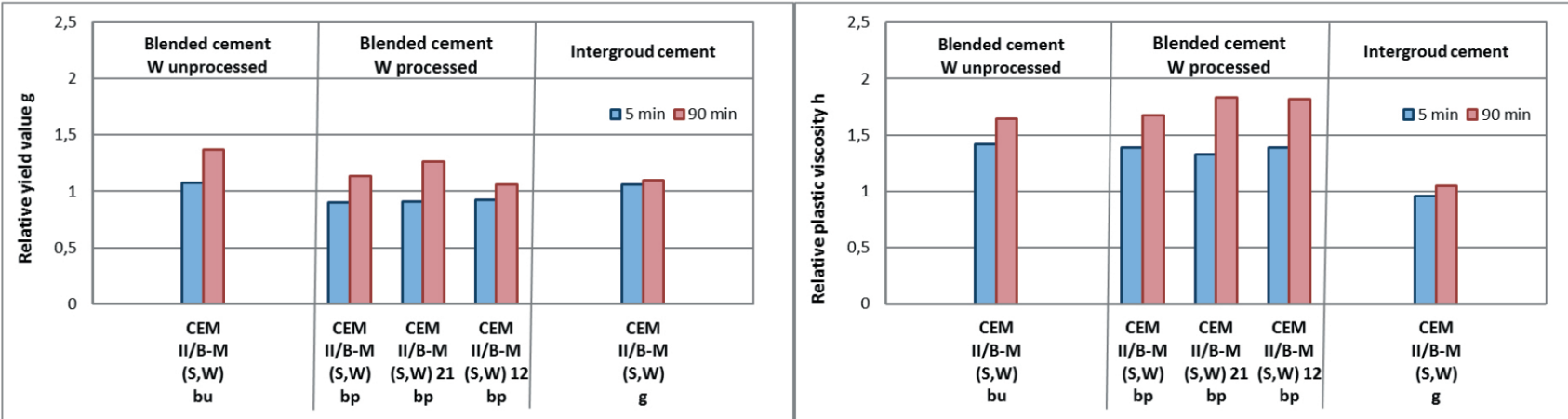

CEM II/B-M (S,W)
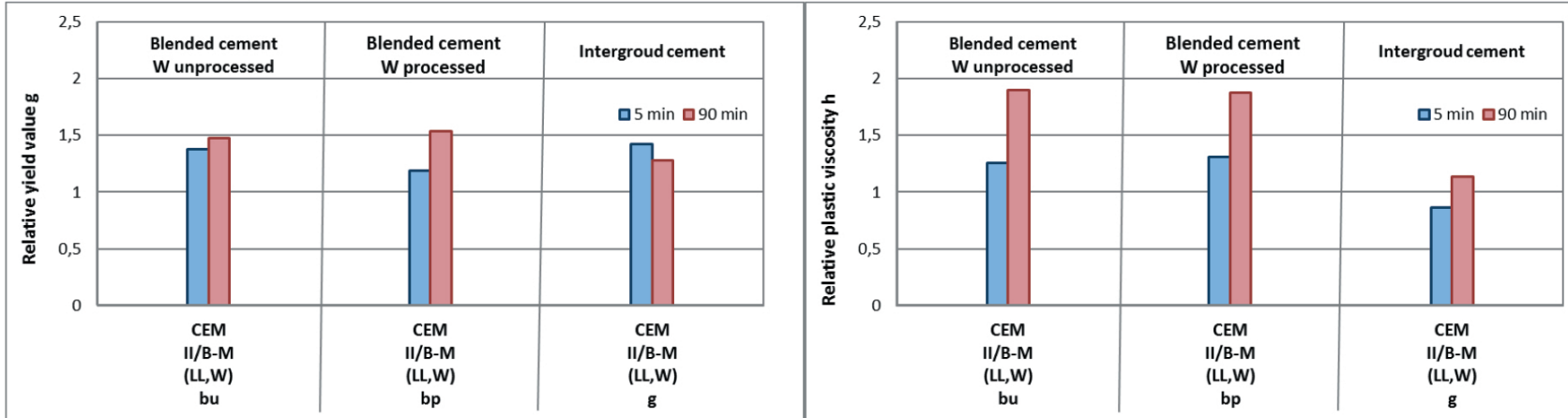

Fig. 2 Relative influence of cements containing calcareous fly ash W on rheological properties of mortars in relations to CEM I mortars, $b u$ - cements produced by blending with raw fly ash $\mathrm{W}, b p$ - cements produced by blending with processed fly ash $\mathrm{W}, g$ - cements produced by intergrinding

cements containing only flay ash W. Changes of rheological properties in time of CEM II/CEM IV (V, W) cement mortars are clearly lower then of CEM II/CEM IV W cement mortars and even of CEM I cement mortars when blended cement with processed $\mathrm{W}$ or interground cement are used. Therefore, studies confirm that the presence of fly ash $\mathrm{V}$ in the cement allows to overcome the negative impact of fly ash $\mathrm{W}$ on the rheological properties of 


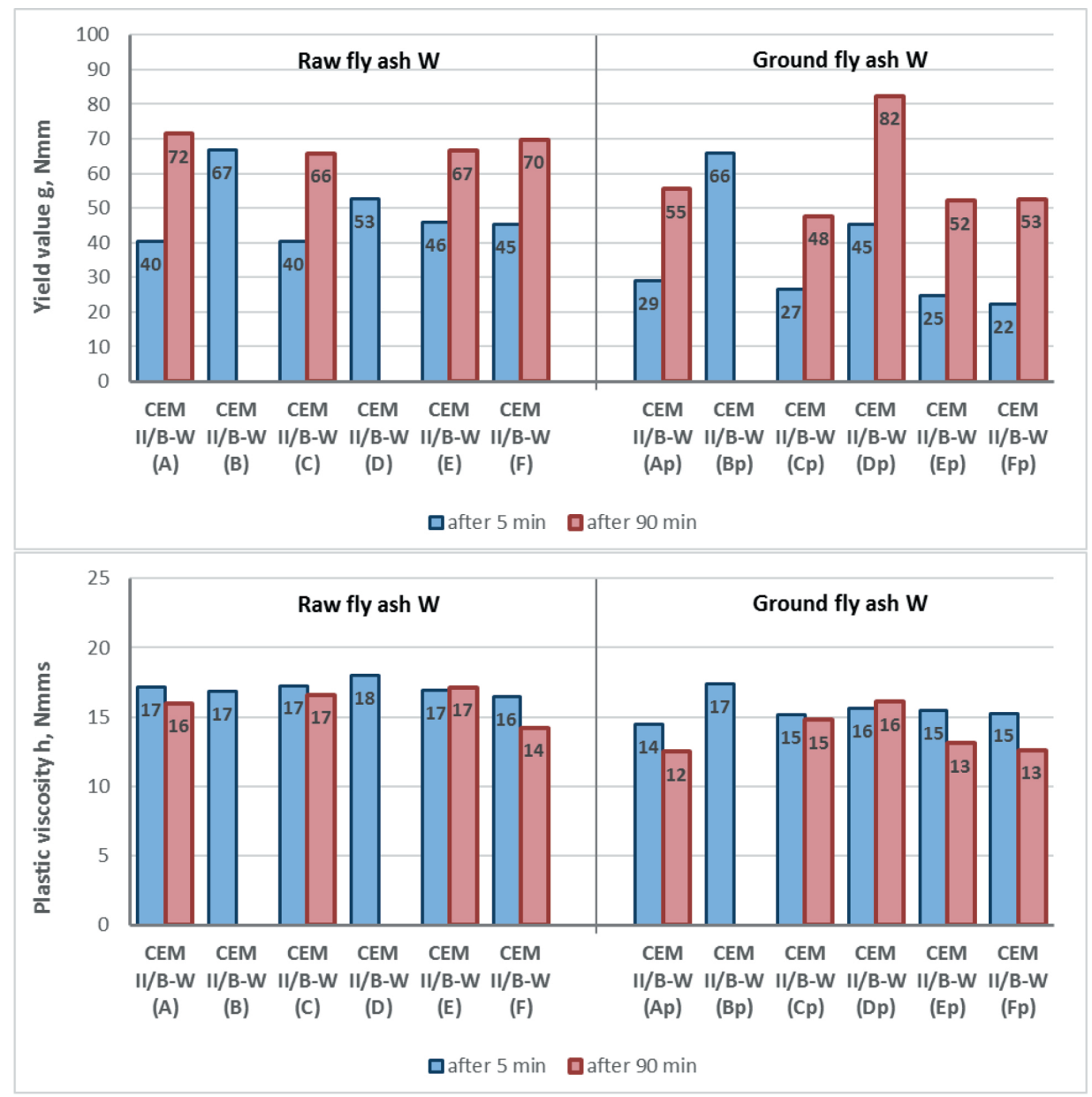

Fig. 3 Influence of calcareous fly ash W batch on rheological properties of mortars made of CEM II/B-W cements
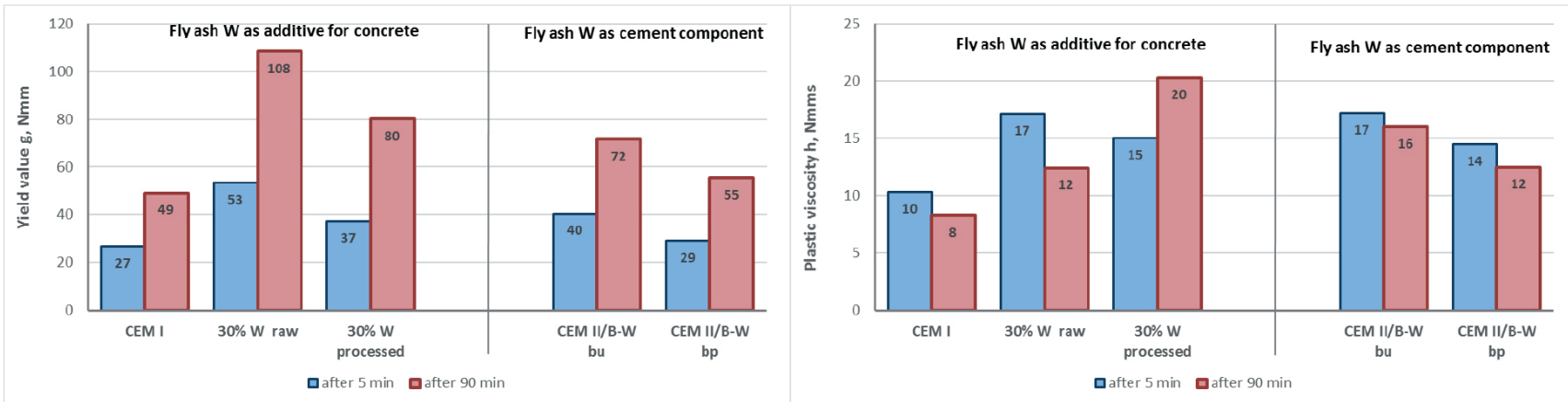

Fig. 4 Rheological properties of CEM II/B-W mortars and mortars with fly ash W as mineral additive for mortar (as part of cement replacement), $b u$ - cements produced by blending with raw fly ash $\mathrm{W}, b p$ - cements produced by blending with processed fly ash $\mathrm{W}$

mortars. It should be noted, that the quantity of $\mathrm{V}$ and $\mathrm{W}$ in cement affects only to a small extend the rheological properties of mortars - yield value $g$ of mortars slightly decreases with increasing $\mathrm{V}$ and $\mathrm{W}$ content in cement (CEM II/A-M (V, W) @ 100 \%, CEM II/B-M (V, W) @ $90 \%$, CEM IV/B-M (V, W) @ 80\%). Therefore, using fly ash $\mathrm{W}$ as cement component together with $\mathrm{V}$ fly ash, it is possible to utilize higher content of $\mathrm{W}$ fly ash overcoming its negative impact on rheology. Considering workability, it is preferable to use the interground or blended with processed fly ash W cements (Fig. 7).

Cements CEM II/A-M (V-W), CEM II/B-M (V-W) and CEMIV/B-M(V-W) do not affect or contribute to reduction of the amount of air in the mortar in relation to the mortars with CEM I cement. Effect of CEM II/CEM IV (V, W) cements and CEM II/CEM IV W cements on the amount 

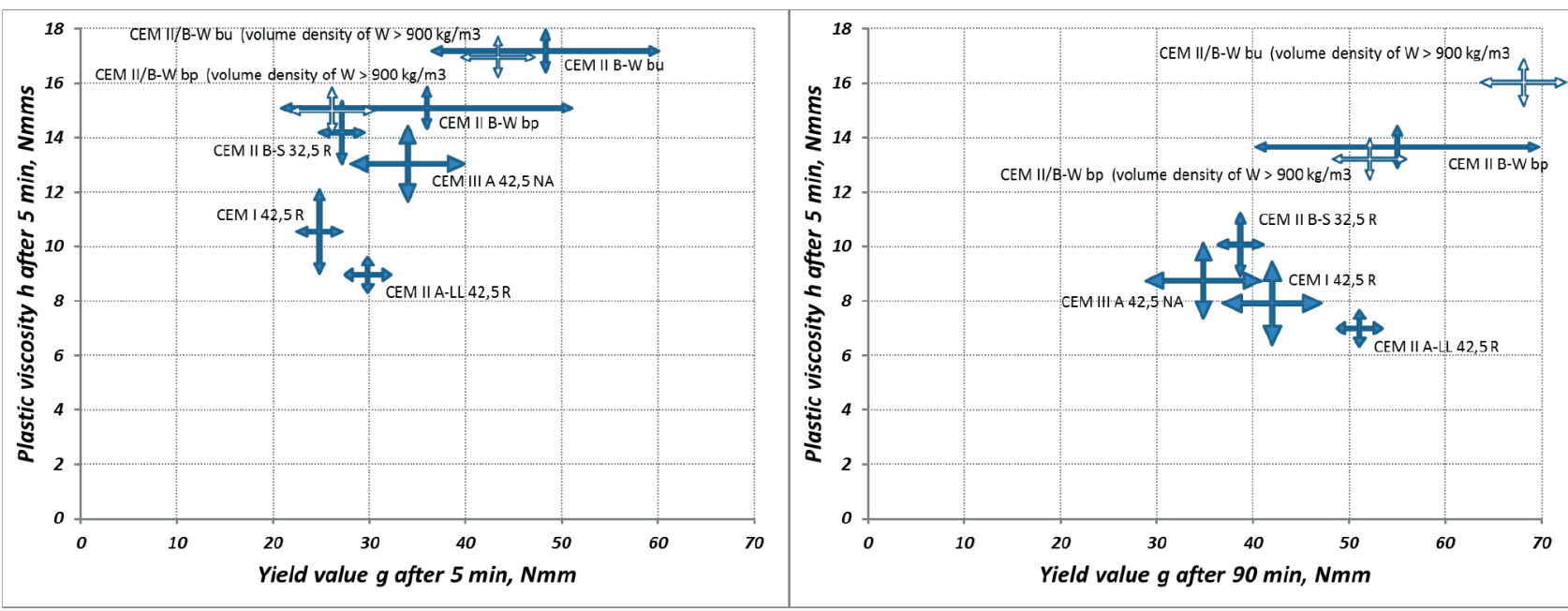

Fig. 5 Variation of rheological properties of mortars made of different cements

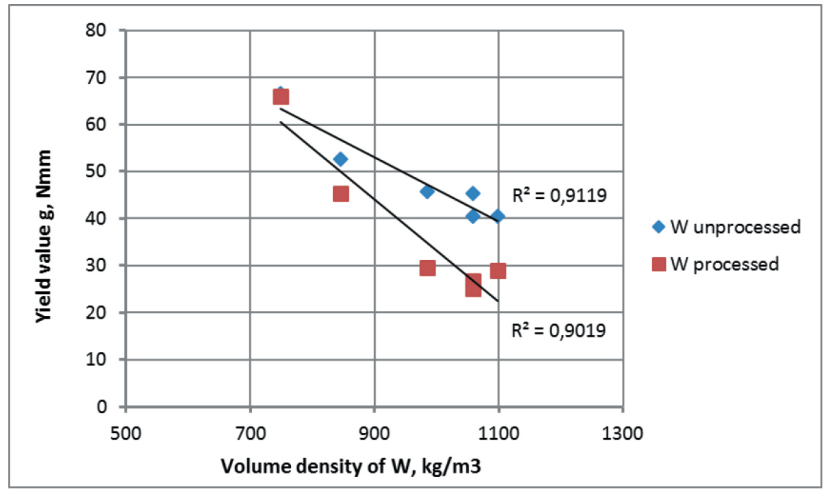

Fig. 6 Yield value g of cement CEM II/B-W mortars vs. volume density of fly ash $\mathrm{W}$

of air in mortar is analogous. Cements with V and W fly ashes are characterized by delayed initial and final setting times in relation to cements with CEM I. Delay of the initial setting time is not explicitly connected to the amount of fly ashes and the cement production process. At the same time, delay of the finial setting time increases with increasing amount of fly ash and is reduced if the cements are obtained by intergrinding. Plastic shrinkage of mortar with cement CEM II/B-M (V-W) is higher than the plastic shrinkage of mortars with CEM II/B-W and CEM I.

Cements containing both $\mathrm{W}$ and $\mathrm{V}$ fly ash and cements CEM I initially are characterized by a similar amount of heat generated during the process of hydration. In the period from 12 to 72 hours the amount of heat generated by cements with $\mathrm{W}$ and $\mathrm{V}$ fly ashes is lower than cements CEM I - in the case of CEM II/A-M (V-W) by approx. $10 \%$, CEM II/B-M (V-W) approx. by $20 \%$, CEM IV/B- M (V-W) by approx. $30 \%$. A method of cement production does not significantly affect the amount of heat generated during 72 hours of hydration.

\subsection{Cements CEM II/B-M (S-W)}

Mortar with cements CEM II/B-M (S-W) obtained by blending or integrounding are characterized by lower yield stress $g$ (higher flow diameter) than the mortars with analogous CEM II/B-W cements and similar (slightly lower or higher) yield stress $g$ in comparison to CEM I mortar. The change of the slag $\mathrm{S}$ to fly ash $\mathrm{W}$ ratio amount in cement CEM II/B-S from $1 / 2$ to $2 / 1$ does not significantly affect the rheological properties of mortars. The increase in the yield stress $g$ in time (decreased of flow diameter) for mortars with blended CEM II/B-M (S-W) cements is lower than for CEM II/B-W mortars and similar or higher than of CEM I mortar. Mortars with blended CEM II/B-S cements have a higher, and mortars with interground cements CEM II/B-S analogous plastic viscosity $h$ as mortar with CEM I. Changing the ratio of slag $\mathrm{S}$ to fly ash $\mathrm{W}$ amount in the cement does not significantly affect the plastic viscosity $h$ of mortar. Plastic viscosity $h$ of mortar with CEM II/B-M (S-W) cement does not change in time. In general, presence of slag $\mathrm{S}$ allows to reduce the negative impact of fly ash $\mathrm{W}$ on the rheological properties of mortars, but to a lesser extent than the corresponding addition of fly ash V. It must be also noted that the ratio of slag $\mathrm{S}$ to fly ash $\mathrm{W}$ amount in the cement in cement affects only to a small extend the rheological properties of mortars. In respect to workability, it is more beneficial to use cements CEM II/B-M (S-W) obtained by blending with processed fly ash W. Cement CEM II/B-M (S-W) obtained by intergrinding has properties only slightly better than cement CEM II/B-W (Fig. 8).

Cement CEM II/B (S-W) does not affect (interground) or contribute to reduction (blended) of the amount of air in the mortar in relation to the mortars with cement CEM I. Effect of cements CEM II/B (S-W) and CEM II/B-W on the 


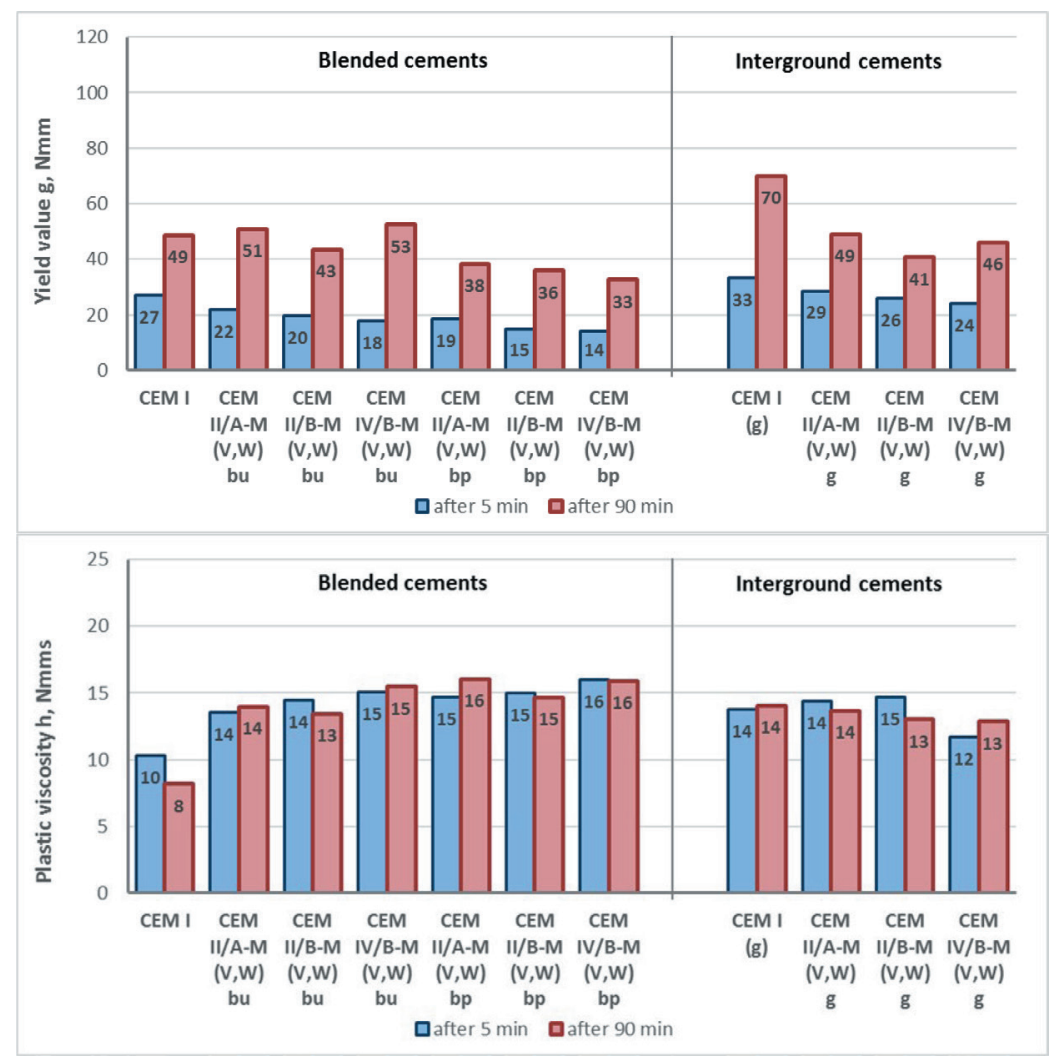

Fig. 7 Rheological properties of mortars made of cements CEM II/A-M (V, W), CEM II/B-M (V, W), CEM IV/B-M (V, W), bu - cements produced by blending with raw fly ash $\mathrm{W}, b p$ - cements produced by blending with processed fly ash $\mathrm{W}, g$ - cements produced by intergrinding
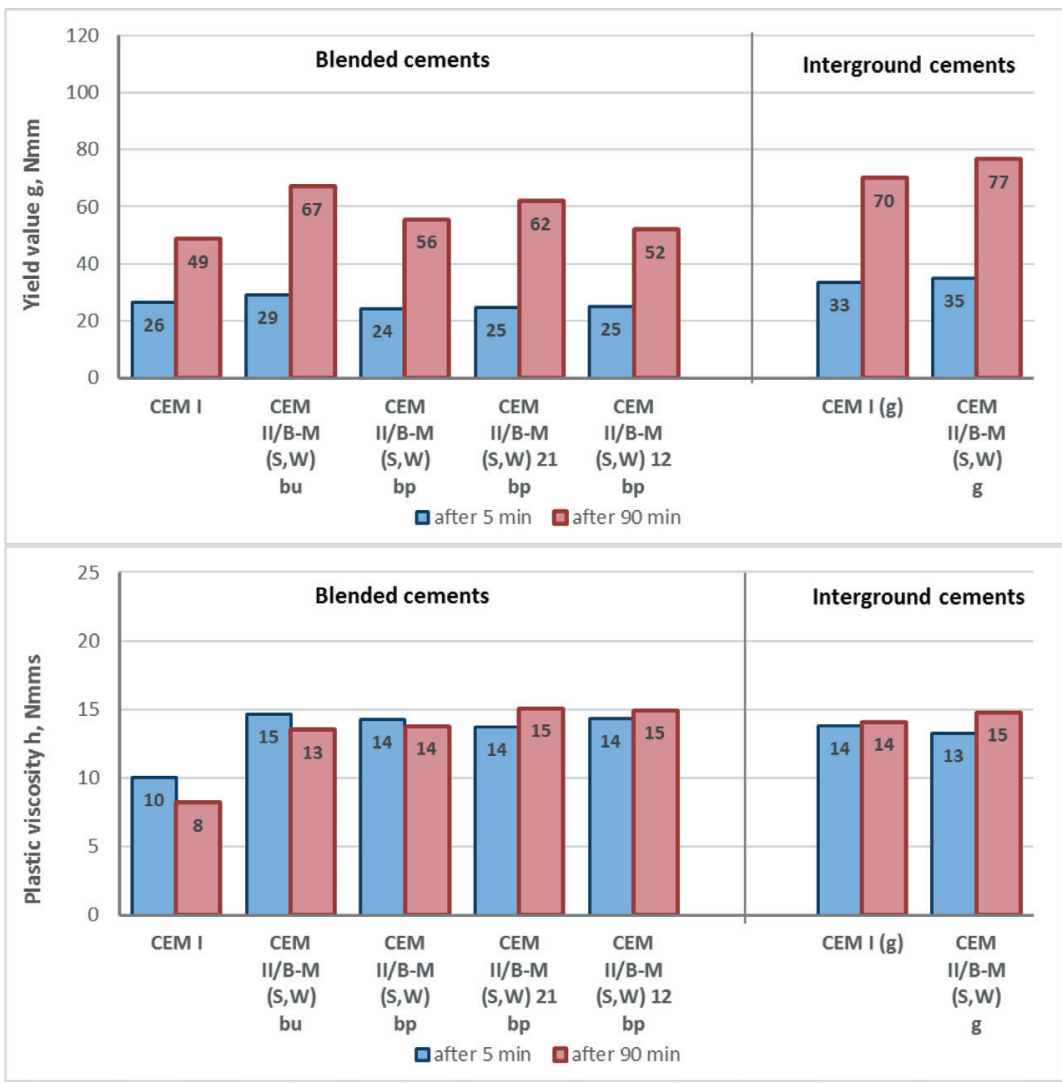

Fig. 8 Rheological properties of mortars made of cements CEM II/B-M (S, W), $b u$ - cements produced by blending with raw fly ash W, $b p$ - cements produced by blending with processed fly ash $\mathrm{W}, \mathrm{g}$ - cements produced by intergrinding 

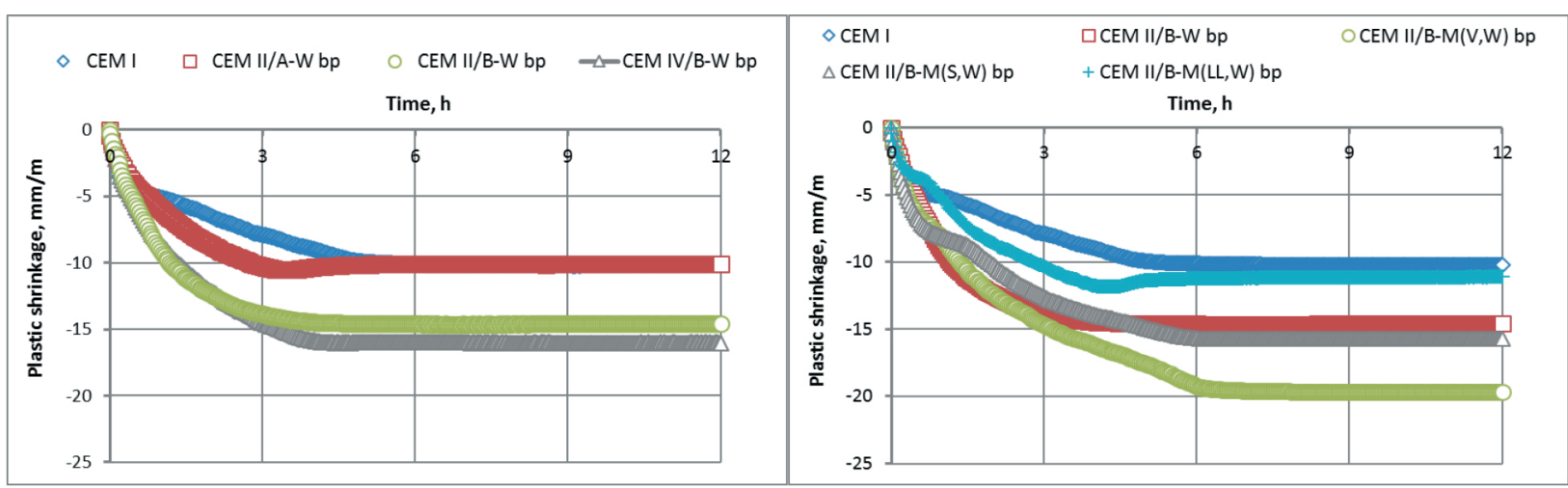

Fig. 9 Plastic shrinkage of mortars made of cements CEM II/A-W, CEM II/B-W, CEM IV/B-W and CEM II/B-M (V, W), CEM II/B-M (S, W), CEM II/B-M (LL, W). Cements produced by blending, fly ash $\mathrm{W}$ processed

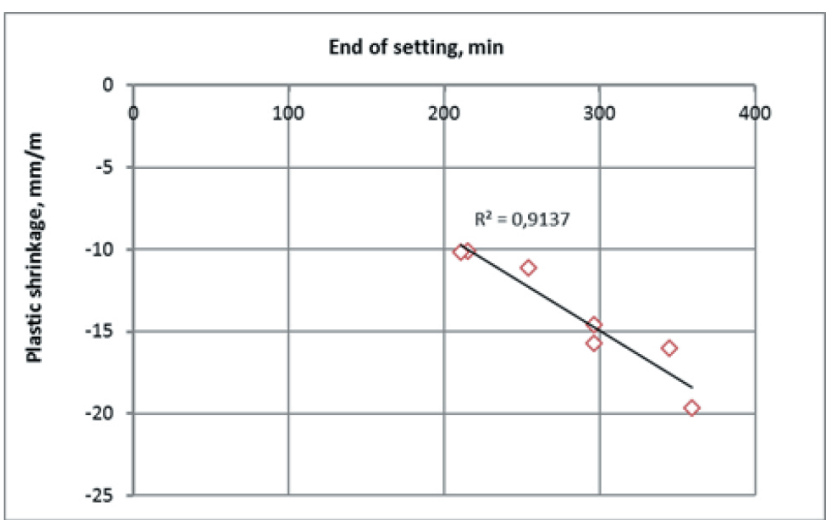

Fig. 10 Plastic shrinkage of cement CEM II/A-W, CEM II/B-W, CEM IV/B-W and CEM II/B-M mortars vs. end of setting of these cements

amount of air in mortar is analogous. Mortars with CEM II/B (S-W) cements in relation to CEM I are characterized by delayed initial and final setting time. This delay depends on quantitative proportions of additives, however, showing no clear trend. In relation to the CEM II/B-W mortars setting time, the initial setting time of CEM II/B (S-W) is similar, but the final setting time can be shorter, especially in the case of blended cement with processed fly ash W. Plastic shrinkage of mortar with CEM II/B-M (S-W) is higher than of mortar with CEM I and similar like mortar with CEM II/B-W (Fig. 9).

Cements CEM II/B (S-W) and cements CEM I initially are characterized by a similar amount of heat generated during the hydration process. In the period from 12 to 72 hours the amount of heat generated by the cements CEM II/B (S-W) obtained by blending is reduced by approx. $15 \%$. In the case of cement CEM II/B (S-W) obtained by intergrinding difference is clearly smaller and amounts to about $7 \%$. Plastic shrinkage of cement CEM II/A-W, CEM II/B-W, CEM IV/B-W and CEM II/B-M mortars vs. end of setting of these cements describes in Fig. 10.

\subsection{Cements CEM II/B-M (LL-W)}

Mortars with cement CEM II/BM (LL-W) obtained by blending have a similar yield stress $g$ (and flow diameters) to mortars with CEM II/B-W and higher yield stress $g$ than mortars with cement CEM I. The increase of the yield stress $g$ in time (decreased flow diameter) of mortar with this cement is higher than in case of both mortar with cement CEM II/B-W and CEM I. The yield stress $g$ of mortar with cement obtained by intergrinding the constituents is higher than of analogous mortar with CEM II/B-W and CEM I. Changes of yield stress $g$ of the mortar in time are smaller than in case of mortar with CEM II/B-W and at the same time higher than of the mortar with CEM I. Mortars with blended cements CEM II/B-M (LL-W) have higher, and mortars with cement interground has analogous plastic viscosity $h$ as mortar with CEM I. Plastic viscosity $h$ of mortars with cement CEM II/B-M (LL-W) slightly increases with time. The presence of limestone LL does not reduce the negative impact of ash (W) on the rheological properties of mortars. Only mortar with cement CEM II/B-M (LL-W) bp (blended with the processed fly ash W) has an initial rheological properties similar to the CEM I and CEM II/B-W mortars, but workability loss of that mortar is clearly higher (Fig. 11).

Cements CEM II/B (LL-W) does not affect (interground) or contribute (blended) to reducing the amount of air in the mortar in relation to the mortars with cement CEM I. In comparison to CEM I, cement CEM II/B (LL-W) has a delayed initial and final setting time. However, in relation to the CEM II/B-W initial and final setting time of CEM II/B (LL-W) are speed up, especially in the case of cement blended with ground fly ash W. The plastic shrinkage of mortar with CEM II/B (LL-W) is slightly higher then mortar with CEM I (Fig. 7). In the period from 12 to 72 hours the amount of heat generated by cement CEM II/B (LL-W) is lesser than CEM I, after 72 hours by approx. 10-15\%. 

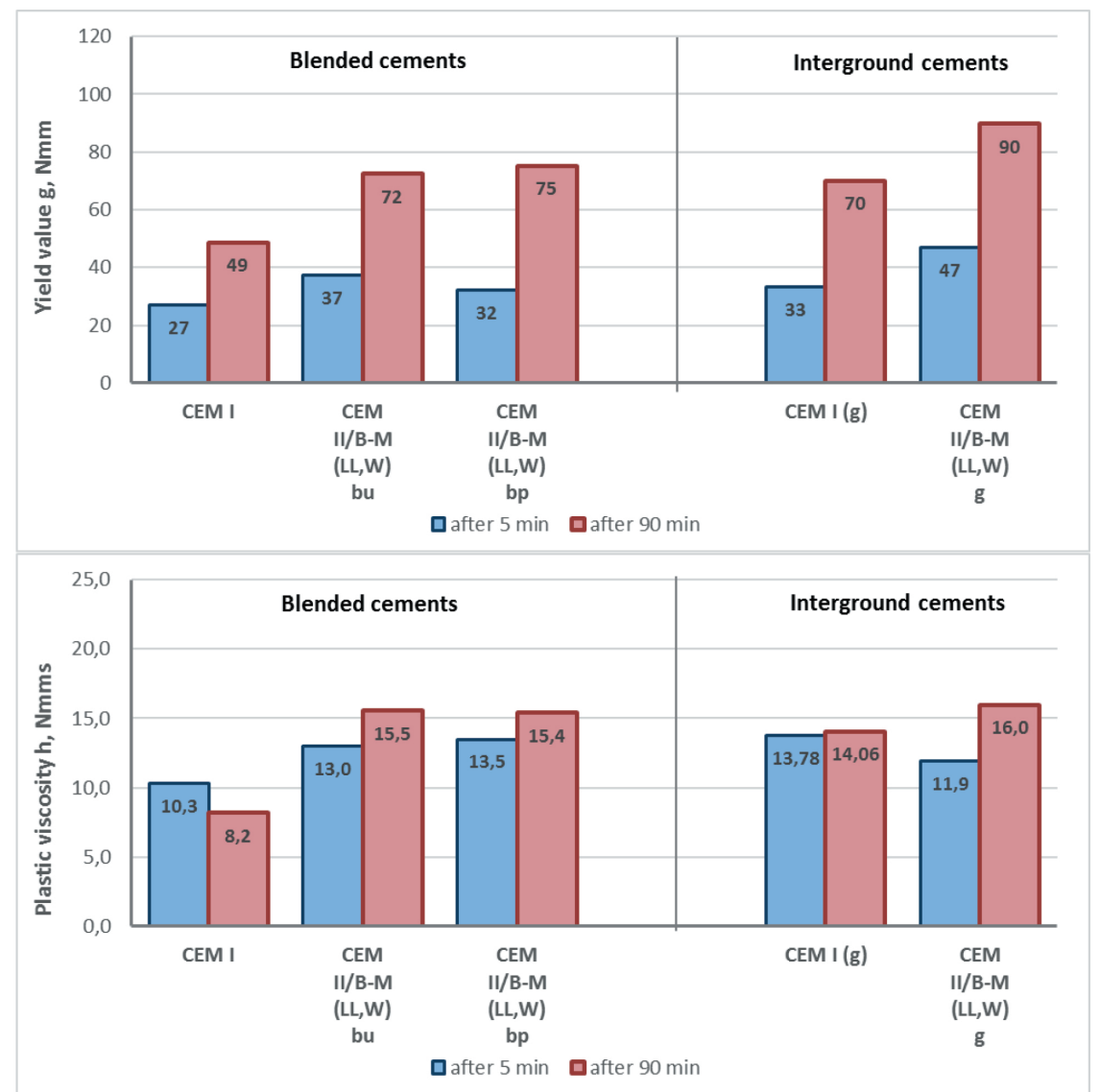

Fig. 11 Rheological properties of mortars made of cements CEM II/B-M (LL, W), $b u$ - cements produced by blending with raw fly ash W, $b p$ cements produced by blending with processed fly ash $\mathrm{W}, g$ - cements produced by intergrinding

\subsection{Effectiveness of various plasticizers type with} cements CEM II/B-W and CEM II/B-M

The influence of plasticizers PL1 and PL2 on rheological properties of the mortars is shown in Figs. 12-13, and on the setting time, air-content of mortars and cement heat of hydration in Table 12.

Fig. 14 shows effectiveness of the plasticizers action PL1 and PL2 in presence of CEM II/B-W and CEM II/B-M cements. It was determined as the ratio of yield stress $g$ of mortars with addition of the plasticizer in amount of $1 / 2$ of the maximal dose to yield stress $g$ of mortar without the admixture. Two plasticisers (PL1 and PL2) differ in chemical base acc. Table 9.

In presence of all cements CEM II containing fly ash W, plasticizers PL1 and PL2 work effectively, regardless of different chemical base, lowering the yield stress $g$ and plastic viscosity $h$ and slowing down the changes of rheological parameters in time. Importantly, the yield stress $g$ of mortars with CEM II/B-W and CEM II/B-M cements containing fly ash $\mathrm{W}$, after addition of plasticizer in amount of $1 / 2$ of maximal dose is usually lower after 5 min than in case of mortars with cement CEM I with analogous plasticizer dose, and for plasticizer PL2 this effect lingers even up to $90 \mathrm{~min}$. Comparing the effects of both plasticizers it can be seen, that PL1 works better with cements CEM II/B-W and CEM II/B-M (V, W), while PL2 better fluidizes mortars with cements CEM II/B-M (S, W) and CEM II/B-M (LL, W). This means that the proper selection of plasticizer requires experimental optimization. Plasticizer PL2 strongly lowers the plastic viscosity of mortars with all of tested cements, most probably due to the air-entraining effect of addition of this admixture (plasticizer PL1 does not exhibit those properties).

Due to the fact that cements with fly ash (W) are characterized by higher water demand, to obtain a set workability for a set w/c ratio, it may be necessary to add a higher dose of plasticizer than in case of CEM I cements. Using a plasticizer one can neutralize the effect of higher workability loss in case of cements with calcareous fly ash (W).

Plasticizer PL1 virtually does not affect (slightly lowers) the aeration, and plasticizer PL2 air-entrains the mortar. This air content in case of mortars with cements containing fly ash $\mathrm{W}$ amounts from 10 to $13 \%$ and is significantly lower than in case of mortars with cement CEM I. 

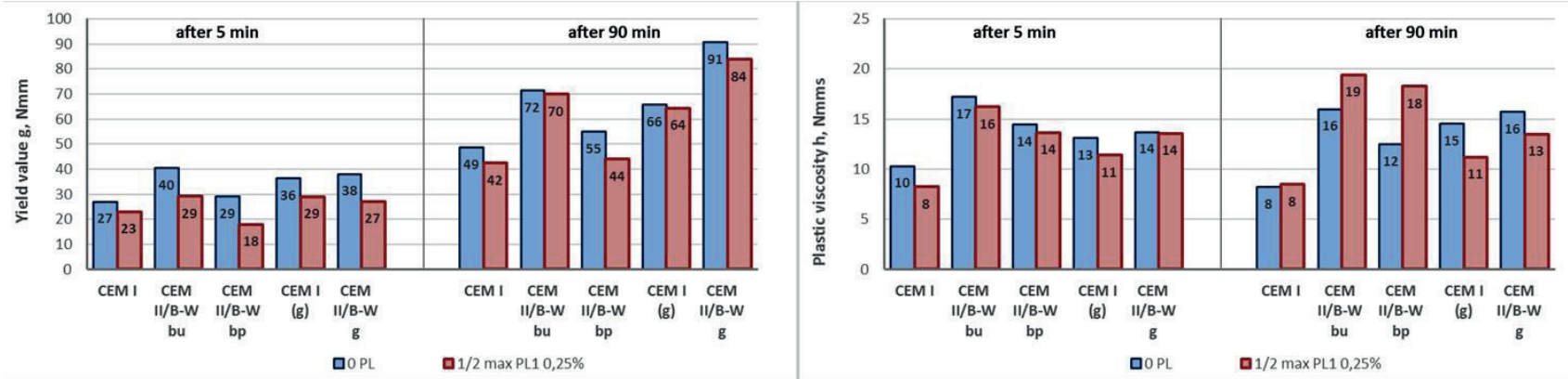

PL1
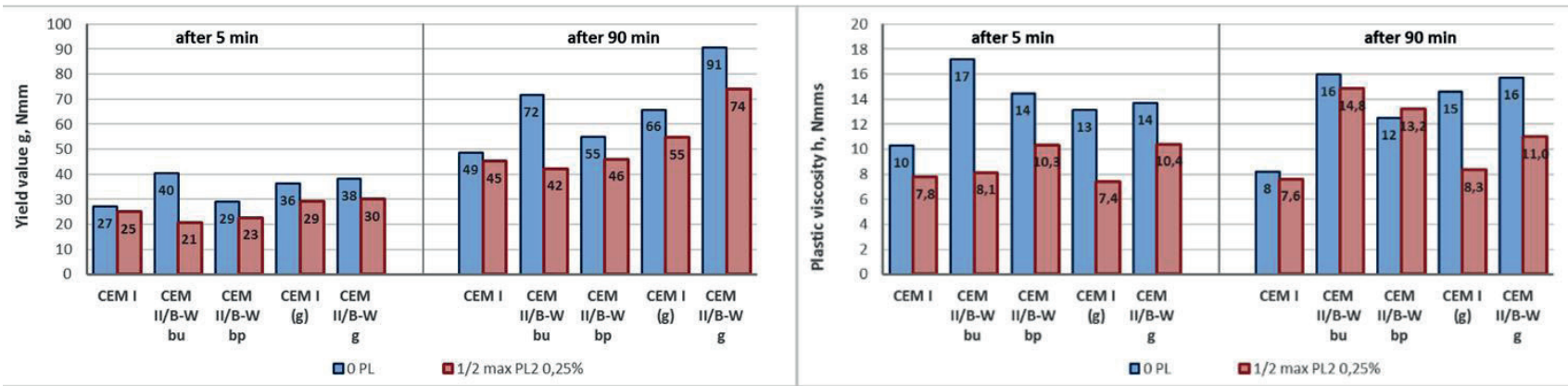

PL2

Fig. 12 Influence of plasticizers PL1 and PL2 addition (1/2 maximum dosage $(0.25 \% \mathrm{C})$ ) on rheological properties of mortars made of CEM II/B-W cements produced by blending (with fly ash W raw $(b u)$ or processed $(b p))$ and by intergrounding $(g)$
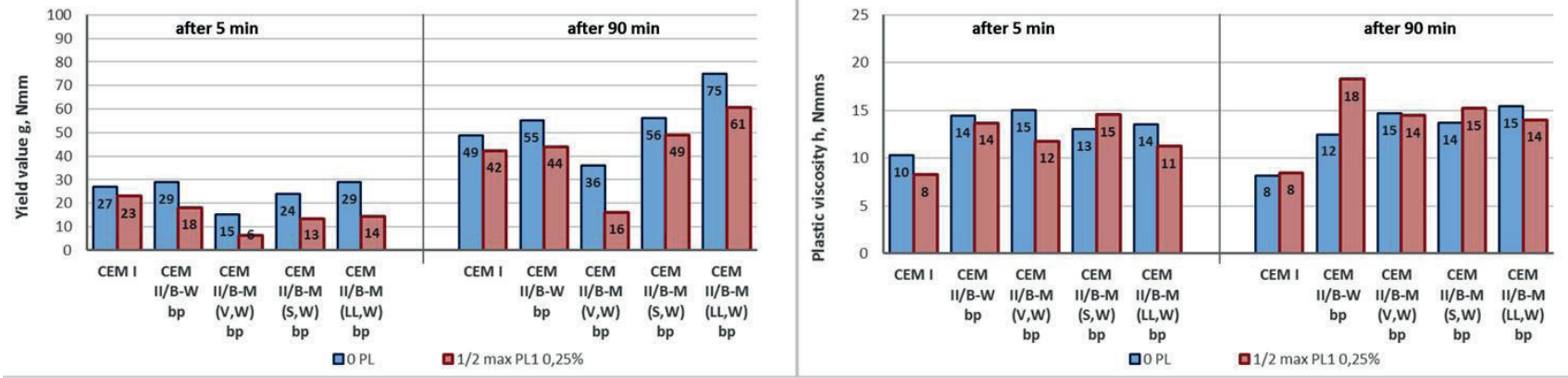

PL1
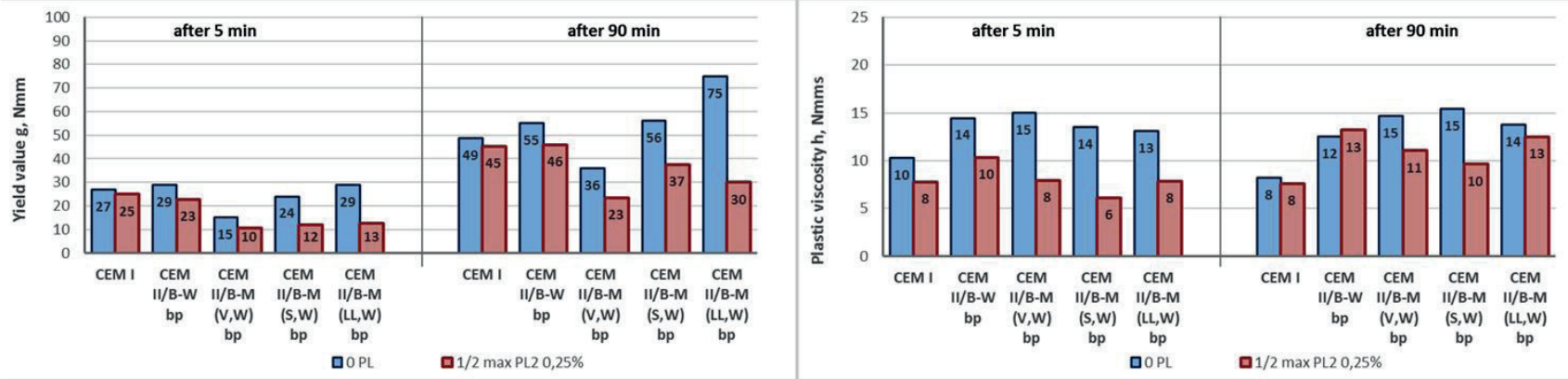

PL2

Fig. 13 Influence of plasticizers PL1 and PL2 addition ( $1 / 2$ maximum dosage $(0.25 \% \mathrm{C}))$ on rheological properties of mortars made of blended CEM II/B-M cements with processed fly ash W 
Table 12 Influence of various plasticizer type on properties of mortars

\begin{tabular}{|c|c|c|c|c|c|c|}
\hline \multirow{2}{*}{ Cement type / Plasticizer type } & \multirow{2}{*}{ Air content, $\%$} & \multirow{2}{*}{$\begin{array}{l}\text { Initial setting } \\
\text { time, min }\end{array}$} & \multicolumn{4}{|c|}{ Hydration heat, $\mathrm{J} / \mathrm{g}$} \\
\hline & & & $12 \mathrm{~h}$ & $24 \mathrm{~h}$ & $48 \mathrm{~h}$ & $72 \mathrm{~h}$ \\
\hline CEM I & 5.4 & 182 & 59.76 & 146.1 & 219.0 & 250.0 \\
\hline CEM I + PL1 & 4.1 & 301 & & & & \\
\hline CEM I + PL2 & 20.5 & 349 & 30.853 & 107.0 & 211.9 & 249.5 \\
\hline CEM II/B-W & 2.5 & 219 & 45.45 & 124.0 & 191.9 & 221.7 \\
\hline CEM II/B-W + PL1 & 2.1 & 385 & & & & \\
\hline CEM II/B-W + PL2 & 10.5 & 444 & 33.774 & 56.946 & 181.8 & 225.6 \\
\hline CEM II/B-M (V, W) & 2.5 & 337 & 39.28 & 117.9 & 180.1 & 206.9 \\
\hline CEM II/B-M (V, W) + PL1 & 2 & 493 & & & & \\
\hline CEM II/B-M (V, W) + PL2 & 13 & 564 & 20.219 & 37.864 & 158.2 & 198.6 \\
\hline CEM II/B-M (S, W) & 2.3 & 247 & 53.43 & 133.5 & 188.2 & 215.2 \\
\hline CEM II/B-M (S, W) + PL1 & 2.4 & 278 & & & & \\
\hline CEM II/B-M (S, W) + PL2 & 11.5 & 322 & 20.219 & 37.864 & 158.2 & 198.6 \\
\hline CEM II/B-M (LL, W) & 2.3 & 260 & 57.23 & 136.0 & 187.5 & 213.9 \\
\hline CEM II/B-M (LL, W) + PL1 & 1.8 & 398 & & & & \\
\hline CEM II/B-M (LL, W) + PL2 & 11 & 461 & 26.198 & 48.485 & 176.9 & 224.12 \\
\hline
\end{tabular}
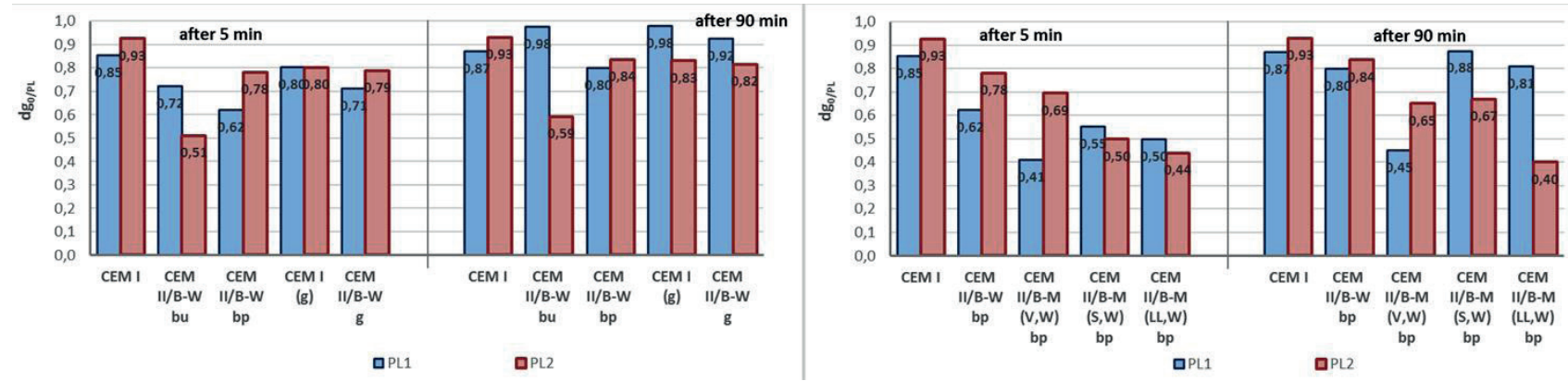

Fig. 14 Relative effect of plasticizers PL1 and PL2 addition ( $1 \frac{1}{2}$ maximum dosage $\left.(0.25 \% \mathrm{C})\right)$ on yield value $\mathrm{g}$ after 5 and 90 minutes of mortars made of cements; a) CEM II/B-W produced by blending (with fly ash W raw (bu) or processed (bp)) and by intergrounding ( $g$ ), and b) CEM II/B-M produced by blending

Plasticizers delay the initial setting time of mortars, but in case of mortars with CEM II/B-M cements containing fly ash $\mathrm{W}$ this effect is clearly lower than in case of CEM I mortars. Plasticizer PL2 lowers the amount of heat generated during hydration in first 12 and 24 hours, however after 72 hours its effect disappears.

\section{Conclusions}

Calcareous fly ash can be successfully used as a main constituent of a wide assortment of cements. The best technological properties are obtained for cements containing siliceous fly ash and to a lesser extent blast furnace slag alongside the calcareous fly ash.

Cements produced by intergrinding of the constituents or blending with fly ash pre-processed by milling, are characterized by acceptable technological properties, not differing significantly from other currently used cements. It is not recommended to use cements obtained by blending with raw calcareous fly ash W.
Calcareous fly ash used for the production of cement should be selected because of its properties. According to conducted tests, this criterion can be the volume density of the ash, which should be at least $900 \mathrm{~kg} / \mathrm{m}^{3}$.

In comparison to concrete mix with CEM I mixes with cements CEM II / A-W, CEM II / B-W, CEM IV / B-W are characterized by worse workability and faster workability loss. These effects are greater the more calcareous fly ash $\mathrm{W}$ is in cement. It should be noted, however, that the negative effect of calcareous fly ash used as an additive for cement on workability is considerably smaller than when it is used as an additive type II.

Mixes with CEM II/A-M (V-W), CEM II/B-M (V-W), CEM IV/B-M (V-W) and CEM II/B-M (S-W) are characterized by higher workability then mixes with CEM II/B-W and similar workability as mixes with CEM I. Using such cements with carefully selected ratio of fly ash W to fly ash $\mathrm{V}$ or to slag $\mathrm{S}$ can reduce or even eliminate the negative influence of fly ash $\mathrm{W}$ on the workability. 
Mixes with CEM II/B-M (LL-W) are characterized by a similar or worse workability and higher workability loss then mixes with CEM II/B-W and CEMI, respectively. From the point of view of improving the conditions of designing the workability, the use of cements containing calcareous fly ash W and ground limestone LL it is not optimal.

Moreover, in comparison to CEM I, cements containing calcareous fly ash (W) or mix of calcareous fly ash (W) and other mineral components - siliceous (V) fly ash, granulated blast furnace slag (S) and limestone (LL) contribute:

- to reduction of the amount of air in the mixes,

- to delaying initial and final setting time mixes,

- to reduction of the amount of heat generated during hydration; during the period of 12-72 hours the amount of heat generated by these cements is smaller by 10 to $20 \%$, and in the case of concretes containing calcareous (W) and siliceous (V) fly ashes by up to $30 \%$,

\section{Reference}

[1] PKN "PN-EN 197-1: 2012 Cement - Part 1: Composition, specifications and conformity criteria for common cements", Polish Committee for Standardization, Warsaw, Poland, 2012.

[2] Neville, A. M. "Properties of Concrete", 5th ed., Pearson Education Limited, London, UK, 2012.

[3] Ramachandran, V. S. "Concrete Admixtures Handbook", 2nd ed., William Andrew, New York, NY, USA, 1996.

[4] Tokyay, M. "Cement and Concrete Mineral Admixtures", CRC Press, Boca Raton, FL, USA, 2016. https://doi.org/10.1201/b20093

[5] Aitcin, P.-C. "Binders for Durable and Sustainable Concrete", CRC Press, Boca Raton, FL, USA.

https://doi.org/10.1201/9781482265767

[6] BGR "Energy Study 2014. Reserves, Resources and Availability of Energy Resources", Federal Institute for Geosciences and Natural Resources (BGR), Hannover, Germany, 2014.

[7] Baran, T., Drożdż, W. "Evaluation of properties of domestic calcareous fly ash and its processing methods", Roads and Bridges, 12, pp. $5-15,2013$.

https://doi.org/10.7409/rabdim.013.001

[8] Dziuk, D., Giergiczny, Z., Garbacik, A. "Calcareous fly ash as a main constituent of common cements", Roads and Bridges, 12, pp. 57-69, 2013

https://doi.org/10.7409/rabdim.013.005

[9] Papadakis, V. G. "Effect of fly ash on Portland cement systems: Part II. High calcium fly ash", Cement and Concrete Research, 30, pp. 1647-1654, 2000. https://doi.org/10.1016/S0008-8846(00)00388-4

[10] Tsimas, S., Moutsatsou-Tsima, A. "HCFA as the fourth constituent in concrete: problems, solutions and perspectives", Cement and Concrete Composites, 27, pp. 231-237, 2005. https://doi.org/10.1016/j.cemconcomp.2004.02.012
- to increase of plastic shrinkage, plastic shrinkage is proportional to the final setting time of the mortars, intensive curing is necessary.

These effects should be taken into account in order to proper and effective use of these cements.

The effectiveness of plasticizers in presence of CEM I cements. CEM II/B-W and CEM II/B-M do not differ significantly or even increases. The method of production of cement does not significantly affect the effectiveness of plasticizers.

\section{Acknowledgement}

Author contributions: All the authors designed experiment, analyzed the results and wrote the paper.

Funding: This research was founded by the EU from the European Regional Development Fund no. POIG 01.01.02.24-005/09 Innovative cementitious materials and concretes made with high calcium fly ashes.

Conflict of interest: The authors have no conflict of interest.

[11] Yazıc1, H. "The effect of silica fume and high-volume Class C fly ash on mechanical properties, chloride penetration and freeze-thaw resistance of self-compacting concrete", Construction and Building Materials, 22, pp. 456-462, 2008 https://doi.org/10.1016/j.conbuildmat.2007.01.002

[12] Gołaszewski, J., Ponikiewski, T., Kostrzanowska, A. "The influence of high calcium fly ash on rheological properties of cement mixtures", In: Non-Traditional Cement \& Concrete IV, Proceedings of the International Conference, Brno, Czech Republic, 2011, pp. 410-419.

[13] Gołaszewski, J., Giergiczny, Z., Cygan, G., Dziuk, D. "The effect of High Calcium Fly Ash on the formation of cement properties with its participation", presented at 13th International Congress on the Chemistry of Cement, Madrid, Spain, July, 3-8, 2011.

[14] Grzeszczyk, S., Lipowski, G. "Popioły lotne i ich wpływ na reologię i hydratację cementów" (Fly ash and their influence on the rheology and hydration of cements), Oficyna Wydawnicza, Wielka Nieszawka, Poland, 2002. (in Polish)

[15] Giergiczny, Z., Synowiec, K., Żak, A. "Suitability evaluation of calcareous fly ash as an active mineral additive to concrete", Roads and Bridges, 12, pp. 83-97, 2013. https://doi.org/10.7409/rabdim.013.007

[16] Giergiczny, Z., Garbacik, A., Ostrowski, M. "Pozzolanic and hydraulic activity of calcareous fly ash", Roads and Bridges, 1, pp. 71-81, 2013. https://doi.org/10.7409/rabdim.013.006

[17] Giergiczny, Z. "Fly Ash as component of cement and concrete", Wydawnictwo Politechniki Śląskiej, Gliwice, Poland, 2013. (in Polish)

[18] Gołaszewski, J., Kostrzanowska, A., Ponikiewski, T., Antonowicz, G. "Influence of calcareous fly ash on rheological properties of cement pastes and mortars", Roads and Bridges, 12, pp. 99-112, 2013. https://doi.org/10.7409/rabdim.013.008 
[19] Knor, G., Glinicki, M. A., Holnicki-Szulc, J., Ossowski, A., Ranachowski, Z. "Influence of calcareous fly ash on the temperature of concrete in massive elements during the first 72 hours of hardening", Road and Bridges, 12, pp. 113-126, 2013.

https://doi.org/10.7409/rabdim.013.009

[20] Gołaszewski, J., Ponikiewski, T. "The effect of calcareous fly ash on selected properties of new generation of concrete", Roads and Bridges, 12, pp. 209-222, 2013. https://doi.org/10.7409/rabdim.013.015

[21] Czopowski, E., Łaźniewska-Piekarczyk, B., Rubińska-Jonczy, B., Szwabowski, J. "Properties of concretes based on cements containing calcareous fly ash", Roads and Bridges, 12, pp. 31-40, 2013. https://doi.org/10.7409/rabdim.013.003

[22] Felekoğlu, B., Türkel, S., Kalyoncu, H. "Optimization of fineness to maximize the strength activity of high calcium ground fly ash Portland cement composites", Construction and Building Materials, 23, pp. 2053-2061, 2009. https://doi.org/10.1016/j.conbuildmat.2008.08.024

[23] Dróżdż, W., Giergiczny, Z. "The resistance of mortars and concrete with calcareous fly ash on alkaline corrosion", Roads and Bridges, 12, pp. 147-158, 2013. https://doi.org/10.7409/rabdim.013.011

[24] Śliwka, A., Domagała, K., Zybura, A. "Evaluation of protective properties of concretes made of cements with calcareous fly ash with respect to reinforcing steel", Roads and Bridges, 12, pp. 237-250, 2013.

https://doi.org/10.7409/rabdim.013.017

[25] Gibas, K., Glinicki, M. A., Nowowiejski, G. "Evaluation of impermeability of concrete containing calcareous fly ash in respect to environmental media", Roads and Bridges, 12, pp. 159-171, 2013. https://doi.org/10.7409/rabdim.013.012

[26] Jóźwiak-Niedźwiedzka, D., Sobczak, M., Gibas, K. "Carbonation of concretes containing calcareous fly ashes", Roads and Bridges, 12, pp. 223-236, 2013. https://doi.org/10.7409/rabdim.013.016

[27] Banfill, P. F. G. "The rheology of fresh mortar", Magazine of Concrete Research, 43(154), pp. 13-21, 1991. https://doi.org/10.1680/macr.1991.43.154.13
[28] Banfill, P. F. G. "The rheology of fresh cement and concrete - a review", In: Proceeding of 11th International Cement Chemistry Congress, Durban, South Africa, 2003, pp. 50-63.

[29] Norberg, J., Peterson, O., Billberg, P. "Effects of a New Generation of Superplasticizers on the Properties of Fresh Concrete", In: Proceeding of Fifth CANMET/ACI International Conference on Superplasticizers and Other Chemical Admixtures in Concrete, Rome, Italy, 1997, pp. 583-598.

[30] Petit, J.-Y., Wirquin, E., Vanhove, Y., Khayat, K. "Yield stress and viscosity equations for mortars and self-consolidating concrete", Cement and Concrete Research, 37, pp. 655-670, 2007. https://doi.org/10.1016/j.cemconres.2007.02.009

[31] Gołaszewski, J., Kostrzanowska-Siedlarz, A., Cygan, G., Drewniok, M. "Mortar as a model to predict self-compacting concrete rheological properties as a function of time and temperature", Construction and Building Materials, 124, pp. 1100-1108, 2016. https://doi.org/10.1016/j.conbuildmat.2016.08.136

[32] Tattarsall, G. H., Banfill, P. F. G. "The Rheology of Fresh Concrete", Pitman Books Limited, Boston, MA, USA, 1983.

[33] PKN "PN-EN 196-1:2006 Methods of testing cement. Determination of strength", Polish Committee for Standardization, Warsaw, Poland, 2006.

[34] PKN "PN-EN 1015-3:2000/A1:2005 Methods of test for mortar for masonry. Determination of consistence of fresh mortar (by flow table)", Polish Committee for Standardization, Warsaw, Poland, 2005.

[35] PKN "PN-EN 1015-7:2000 Methods of test for mortar for masonry. Determination of air content of fresh mortar", Polish Committee for Standardization, Warsaw, Poland, 2000.

[36] PKN "PN-EN 480-2:2008 Admixtures for concrete, mortar and grout. Test methods. Determination of setting time", Polish Committee for Standardization, Warsaw, Poland, 2008.

[37] Schleibinger Testing System "Shrinkage Code", [online] Available at: http://www.schleibinger.com/cmsimple/en/?Shrinkage:Shrinkage-Cone [Accessed: 28 February 2017] 